\title{
Simultaneous planar measurement of droplet velocity and size with gas phase velocities in a spray by combined ILIDS and PIV techniques
}

\author{
Yannis Hardalupas ${ }^{1}$, Srikrishna $\mathrm{Sahu}^{2}$, Alex M.K.P. Taylor ${ }^{3}$, Konstantinos Zarogoulidis ${ }^{4}$ \\ 1: Dept. of Mechanical Engineering, Imperial College, London, UK \\ 2: Dept. of Mechanical Engineering, Imperial College, London, UK \\ 3: Dept. of Mechanical Engineering, Imperial College, London, UK \\ 4: Dept. of Mechanical Engineering, Imperial College, London, UK \\ Tel: $+44(0) 2075947057$ \\ Email: y.hardalupas@imperial.ac.uk
}

\begin{abstract}
A new approach for simultaneous planar measurement of droplet velocity and size with gas phase velocities is reported, which combines the out-of-focus imaging technique 'Interferometric Laser Imaging Droplet Sizing' (ILIDS) for planar simultaneous droplet size and velocity measurements with the in-focus technique 'Particle Image Velocimetry' (PIV) for gas velocity measurements in the vicinity of individual droplets. Discrimination between the gas phase seeding and the droplets is achieved in the PIV images by removing the glare points of focused droplet images, using the droplet position obtained through ILIDS processing. Combination of the two optical arrangements can result in a discrepancy in the location of the centre of a droplet, when imaging through ILIDS and PIV techniques, of up to about $1 \mathrm{~mm}$ which may lead to erroneous identification of the glare points from droplets on the PIV images. The magnitude of the discrepancy is a function of position of the droplet's image on the CCD array and the degree of defocus, but almost independent of droplet size. Specifically, it varies approximately linearly across the image along the direction corresponding to the direction of propagation of the laser sheet for a given defocus setting in ILIDS. The experimental finding is supported by a theoretical analysis, which was based on geometrical optics for a simple optical configuration that replicates the essential features of the optical system. The discrepancy in the location was measured using a monodisperse droplet generator and this was subtracted from the droplet centres identified in the ILIDS images of a polydisperse spray without 'seeding' particles. This reduced the discrepancy between PIV and ILIDS droplet centres from about $1 \mathrm{~mm}$ to about $0.1 \mathrm{~mm}$ and hence increased the probability of finding the corresponding fringe patterns on the ILIDS image and glare points on the PIV image. In conclusion, it is shown that the proposed combined method can discriminate between droplets and 'seeding' particles and is capable of two-phase measurements in polydisperse sprays.
\end{abstract}

Keywords: ILIDS, glare points, wavelet transform, centre discrepancy 


\section{Introduction}

In liquid-fuelled combustion, the process of air-fuel mixture formation is influenced by air entrainment, and coupling between the dispersed and continuous phases. The interaction of a spray with the airflow field redistributes the droplets according to differences in droplet inertia, momentum and drag. Because of the different relaxation times of droplets of different sizes, interaction with various scales of eddies in a turbulent flow field can lead to cluster formation (Sanchez et al. 2000; Zimmer et al. 2003). "Centrifuging" of larger droplets out of eddies may lead to local droplet void regions resulting in large temporal and spatial variation of the distribution of droplets and possibly, also, local fuel vapour concentration. In this context, experiments on spray flows are essential not only for understanding the mechanisms of droplet-gas flow interaction but also for the development and evaluation of spray models. For a spray-laden gas, the turbulent kinetic energy equation for the dispersed phase contains terms which include correlations of droplet concentration and velocity fluctuations of fluid and/or droplets and droplet-fluid velocity correlations (Hardalupas and Horender 2003). They represent an 'extra' source or sink of turbulent kinetic energy in the fluid and depict the interaction, which needs to be modelled successfully. In this regard experiments need to characterize not only the relative velocity between the droplet and gas phases and associated spatial correlations but also the droplet size simultaneously, which is the ultimate aim of the present research.

Optical measurements in two phase flows are difficult because of the need to discriminate between the two phases. In planar, image-based techniques with laser illumination, this is usually done either by acquiring images of both phases in a single camera followed by image processing to separate the phases or by using two cameras each arranged to acquire images from individual phases in some way. A popular approach is to tag the gas phase seeding with a fluorescent dye in conjunction with an adequate optical filter that attenuates the Mie scattered signal from the spray droplets. For example, Lindken and Merzkirch (2002) used only one camera for bubbly flow measurement based on a combination of PIV with fluorescent tracer particles and shadowgraphy and digital phase separation with a masking technique. Similarly, Rottenkolber et al. (2002) acquired consecutive images from the spray alone and of the induced gas flow alone using fluorescent seeding particles to trace the gas phase. They described two different algorithms for phase discrimination known as 'mask' and 'peak' separation techniques. In order to avoid the presence of the two phases on the same image, Kosiwczuk et al. (2005) tagged both phases, instead of only one, with two different fluorescent dyes. Two cameras 
were used one for each phase along with suitable optical filter set. They could obtain simultaneous and independent velocity fields of the two phases by processing each image containing only one phase by standard Particle Image Velocimetry (PIV) or Particle Tracking Velocimetry (PTV) algorithms. Since knowledge of the droplet size is equally important as the droplet and gas velocity, PIV alone is not sufficient for the task. Classical single-point techniques, though reliable, cannot easily identify issues such as preferential concentration and coherent flow structure identification in a spray. The fluorescence approach is always associated with the possibility of cross-contamination due to Mie scattering, which needs to be quantified, and moreover it is relatively expensive to use. Specifically it is unsuitable with respect to the work reported here (using a defocused technique for planar droplet sizing and velocity) in which finding the droplet centre location is a crucial factor for discriminating between the two phases. The literature on two phase planar measurements in sprays does not yet provide either extensive information on droplet size-velocity correlation (though reporting the velocities of the dispersed and the carrier phases) or the gas phase velocity (providing only size and velocity of the dispersed phase). To the authors' knowledge, the optical set-up mentioned below is the first one to be reported which is capable of simultaneous planar measurement of both droplet and gas properties with the droplet-gas velocity spatial correlation calculated conditional on droplet size.

The purpose of this paper is to describe an optical instrument for the simultaneous planar (or whole field) measurement of droplet size and velocity along with the gas velocity in a spray by combining the 'out-of-focus imaging' technique ILIDS (Interferometric Laser Imaging for Droplet Sizing) for planar droplet size and velocity measurements with PIV for gas phase velocity measurements (Fig.1). ILIDS (also known as Interferometric Particle Imaging, IPI) is an optical technique for instantaneous measurements of the spatial distribution of individual droplet size and velocity in polydisperse sprays. It makes use of the spacing of the interference fringes formed due to reflected and first order refracted scattered light from individual droplets, when collected by 'defocused optics', to determine the droplet size (Glover et al. 1995). The incorporation of image compression optics by Maeda et al. (2000) reduces fringe overlapping in dense sprays and avoids the complexity of the evaluation of fringe spacing (Damaschke et al. 2005) and thus extends the applicability of ILIDS to relatively denser sprays. The advantage of the present technique lies in the fact that the position of droplets in a spray, obtained by ILIDS beforehand, helps in identifying the images of the same droplets in the focused PIV image, thus making it possible to remove these from the PIV image. In this way the PIV image retains only 'seeding' particles, which follow the gas phase flow, and can be processed to 
obtain the gas velocity in the vicinity of each droplet. The difficulty with the combined technique is the presence of a discrepancy in droplet centres when calculated independently through ILIDS and PIV images. The problem of droplet centre discrepancy can be dealt in several ways although its existence is rarely recognized in the literature. One exception is Kurosawa et al. (2002), while describing the method of combining ILIDS with Laser Induced fluorescence (LIF) for simultaneous vapour concentration measurement along with droplet properties, noticed the discrepancy of droplet position in the two images. The reason behind the occurrence of the droplet positioning error was attributed to "the accuracy of position calibration caused by the use of two cameras for each measurement". Two other exceptions are Palero et al. $(2005,2007)$ who used a holographic technique to record the defocused image fields of the dispersed phase, collecting light at a scattering angle of $90^{\circ}$, with the convenience of using a single camera. They were able to reconstruct the particle image in any defocused, or in the best-focused, plane by numerically propagating the defocused image, thus implicitly eliminating any discrepancy in particle position in addition to avoiding the inconvenience of fringe overlapping. In the current work, we use a scattering angle of less than $90^{\circ}$ - with the advantage of a stronger signal using vertically polarized light - and show that the discrepancy in droplet position is inevitable in the use of the ILIDS technique and so it needs to be quantified.

The objectives of the present paper are to show the cause of this discrepancy, quantify the error and propose a method of correction. The paper starts with a description of the combined ILIDS/PIV instrument, followed by a theoretical analysis based on the optical configuration. The results are compared qualitatively with experimental results. The application of the proposed method, including the image processing algorithm, is demonstrated at first for a stream of mono size droplets produced by a custom-made droplet generator. This was done in order to avoid the complexity arising from a polydisperse spray and its much higher droplet number density. The droplet generator is, secondly, evaluated in the presence of a slow co-flowing air stream, seeded with particles for PIV measurements. Results corresponding to the spray without any seeding surrounding it are presented and, finally, preliminary results in sprays with seeding are shown.

\section{Experimental set up for combined ILIDS with PIV measurements}

The fundamental principle of combining the optical arrangements of ILIDS with PIV is considered by using geometrical optics. When a transparent spherical water droplet in a flow field is illuminated by a 
coherent laser source (Fig 1a), the reflected and first order refracted light scattered from the droplet dominate in the forward scatter region for angles between around $30^{\circ}$ to $80^{\circ}$. On a defocused plane the reflected and refracted rays interfere to produce parallel fringes (Glover et al. 1995). The number of fringes present in each of the imaged fringe patterns and the fringe spacing is proportional to the diameter. The characteristic interferogram is observed at a defocused plane with a far field arrangement of receiving optics (Kawaguchi et al. 2002) through camera 1 (Fig. 1a). The defocusing is achieved and controlled by using a pair of cylindrical lenses placed in between the collecting lens (lens 1) and camera 1.

For the purpose of characterizing simultaneously the velocity of the air flow in the vicinity of individual droplets, the air surrounding the spray is seeded with particles and the viewing area is imaged for PIV measurements. This is achieved by splitting a part of the incoming scattered light using a beam splitter and collecting it through a second camera, camera 2 (Fig. 1a). With this optical system, bright spots called glare points, corresponding to focused reflected and refracted rays, appear in camera 2. Hence, the same droplet is imaged as a rectangular region with a superimposed fringe pattern on the ILIDS camera and as distribution of two glare points on the PIV image. The defocused images from 'seeding' particles (if not liquid droplets) appear on the ILIDS camera, but without any superimposed fringes. Usually, for both cases, the centre of a droplet is assumed to be the geometrical centre of the fringe pattern/glare points, but this need not be the same as the actual droplet centre.

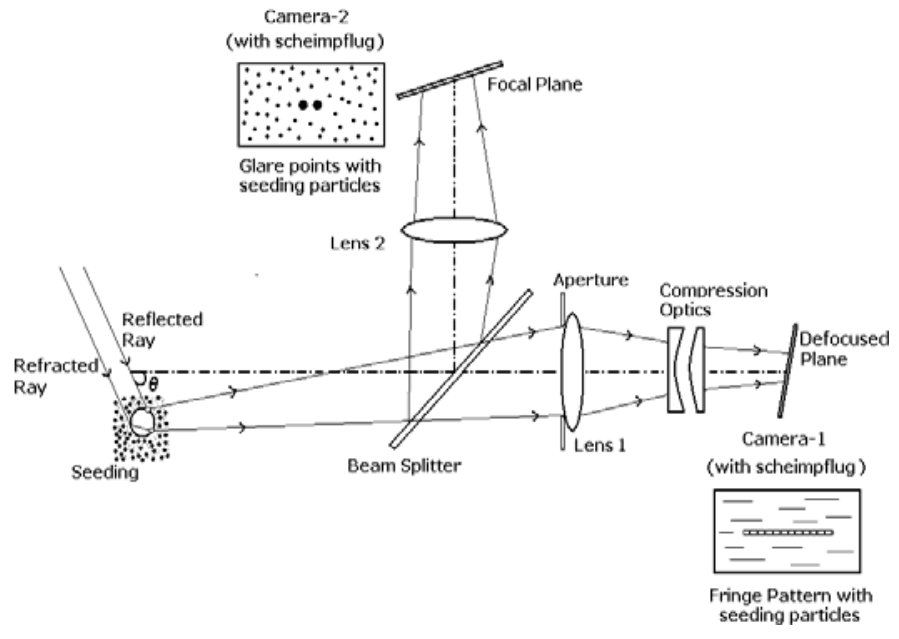

(a)

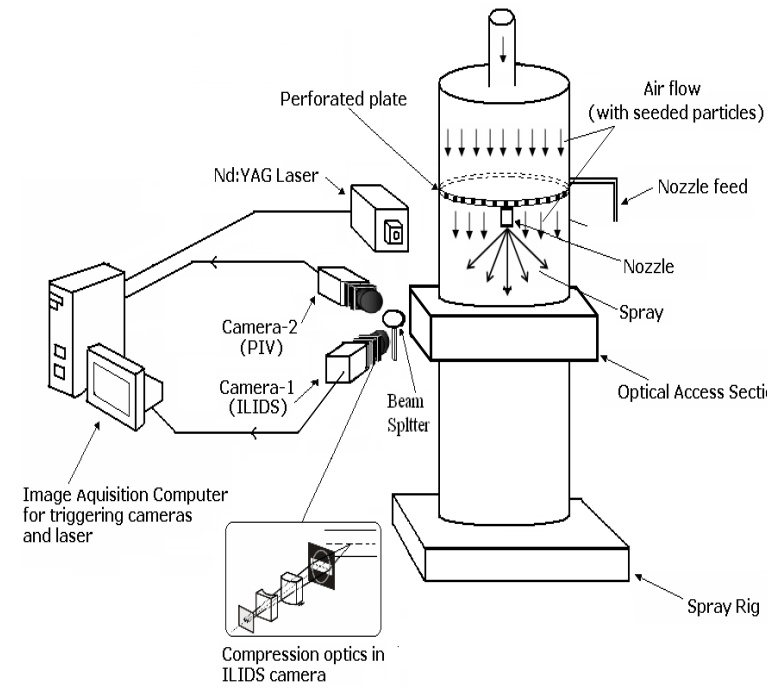

(b)

Fig. 1 (a) Principle of the combined ILIDS and PIV technique (b) Experimental set-up 
An overview of the experimental rig is shown in Figure 1b. The present work employed a spray dryer rig for two phase measurements, the details of which can be found in Kavounides (2006). The rig allowed co flowing air to enter from the top in the annulus around the atomiser, which was a custombuilt air-assisted nozzle placed on the centreline of the cylindrical chamber with diameter of $0.5 \mathrm{~m}$. It produced a solid cone spray with a Sauter Mean Diameter (SMD) of the order of 150-200 $\mu \mathrm{m}$ at liquid feed rates of the order of $1.4-1.6 \cdot 10^{-3} \mathrm{~kg} / \mathrm{s}$ and air feed rate of the order of $0.12 \cdot 10^{-3} \mathrm{~kg} / \mathrm{s}$. The co flowing air was seeded with aluminium oxide particles (diameter range 1-5 $\mu \mathrm{m}$ ) before entering the rig. The co flowing air flow rate, carrying the seeding particles, was $4 \cdot 10^{-3} \mathrm{~kg} / \mathrm{s}$, resulting in areaaveraged air velocity $1.7 \times 10^{-2} \mathrm{~m} / \mathrm{s}$ around the spray.

A frequency-doubled, double pulse Nd:YAG laser $(120 \mathrm{~mJ} /$ pulse at $532 \mathrm{~nm}$; New Wave Research) was used to illuminate the flow. The laser sheet was generated with a cylindrical lens (focal length $=-50 \mathrm{~mm}$ ) and a spherical lens (focal length $=+200 \mathrm{~mm}$ ) respectively. Two identical cameras were used (PCO; Sensicam QE, 12bit, 1040×1376) and positioned on the same side of the laser sheet. Two identical lenses (Nikon; $135 \mathrm{~mm}$ focal length) were used to collect the scattered light from the droplets. Defocusing of the image in camera 1 was achieved, as mentioned before, by a pair of cylindrical lenses $(+50 \mathrm{~mm}$ and $-50 \mathrm{~mm}$ focal length). The scattered light from droplets was divided into two parts by using a pellicle beam splitter of thickness $2 \mu \mathrm{m}$ (Fig. 1b) to avoid formation of ghost images. The light refracted through the pellicle beam splitter was directed at the defocused plane for measurements with ILIDS, while the reflected light was directed for PIV measurements. The purpose behind this configuration is that the reflected light from the beam splitter is more sensitive to the alignment of the beam splitter than the refracted one. Since the ILIDS camera was usually operated with maximum aperture, the problem of image distortion would have been more pronounced if the reflected light were used. Because of the issues related to optical aberrations of ILIDS images, both cameras were adjusted to provide a field of view of approximately $10 \times 15 \mathrm{~mm}$, which is comparatively small with respect to that of usual PIV system operation. The resolution was approximately $10 \mu \mathrm{m} /$ pixel in both directions and the magnification was about 0.60 , for both cameras. In all experiments, the scattering angle was set at $\theta=69^{\circ}$, which is the optimum scattering angle for refractive index of 1.33 (water in air) for maximum interference between refracted and reflected rays for ILIDS operation with a vertically polarized laser sheet. Although the use of horizontally polarized light gives the highest visibility at a scattering angle of $90^{\circ}$, and would also obviate the need for the Scheimpflug condition, the scattered intensity is smaller by about 10 to 100 times than for the forward scatter configuration 
(Damaschke et al. 2005). Hence this option is not always convenient. The collecting angle was set to $6.35^{\circ}$, resulting in a resolution of 5.28 [ $\mu \mathrm{m} /$ fringe] for the ILIDS system. Experiments were first performed with a custom built droplet generator, producing a single stream of monosized droplets. For this purpose, the droplet generator was supported inside the rig and seeded air was allowed to flow around it. The pinhole size at the exit of the monodispersed droplet generator was $100 \mu \mathrm{m}$. The injection pressure was set at 1.0 bar, while the water flow rate was $6.0 \mathrm{~cm}^{3} / \mathrm{min}$. The resonance frequency of the piezoelectric elements of the generator was set to $20 \mathrm{kHz}$. Under this condition, the diameter of the droplets was approximately $212 \mu \mathrm{m}$ with an accuracy of 97\% (Pergamalis, 2002).

The field of view of this system was not parallel to the CCD array of either camera due to the requirement of imaging at a scattering angle $\theta$, which is different than $90^{\circ}$. Hence, in order to avoid varying the degree of focus/defocus across the CCD array, both the cameras were aligned under the Scheimpflug condition similar to Sugimoto et al. (2006). The Scheimpflug condition is not essential for ILIDS $^{1}$ but its use results in uniform length of the droplet fringe patterns, thus making the fringe length detection more convenient. In the present case, because of relatively smaller viewing area, negligible variation in magnification ( 0.5676 to 0.5854$)$ was observed across the image. Throughout the text, the term "focused plane" refers to the PIV image (which would not be true without application of the Scheimpflug criterion), while the "defocused plane" refers to the ILIDS image. The camera calibration is a crucial factor for the accuracy of the proposed optical arrangement. To ensure both cameras imaged the same area, a calibration plate with equally spaced "crosses" was used. Note that the calibration was performed with both cameras in focus. The location of the centres of the crosses were determined with sub-pixel accuracy and mapped to the respective pixels by fitting a mapping function (cubic polynomial), the coefficients of which were obtained through linear least squares approximation. The accuracy in calibration was $\pm 15 \mu \mathrm{m}$ and $\pm 4 \mu \mathrm{m}$ in horizontal and vertical directions respectively. Thus, the real position in space could be obtained, given a location on the image.

\section{Theoretical prediction of droplet centre discrepancy}

The optical configuration described in the previous section results in a difference in the location of the centre of any given droplet in the two images obtained by ILIDS and PIV. Figure 2a shows a simplified

\footnotetext{
${ }^{1}$ but is vital for PIV because the image has to be captured in the 'in focus' condition.
} 
ray diagram in which the direction of the light sheet is shown by an arrow and we assume that the droplet centre (point ' 0 ') is co-linear with the corresponding glare points (points ' 1 ' and ' 2 '). A similar imaging system can also be found in Damaschke et al. (2005), though their focus was on droplet image size in the defocus plane rather than the discrepancy in droplet centre between focus and defocus images. In figure $2 \mathrm{a}$, the positions of the droplet normal to, and within, the plane of the laser sheet are denoted by the two Cartesian axes $x$ and $z$. The analysis does not consider the droplet position normal to the $x z$ plane i.e. along the $y$ direction. We expect this limitation to have minor influence over the result. For simplicity, we represent the images on the ILIDS and PIV CCD arrays as being formed by a single lens instead of, as in the experiment, by two independent lenses (Fig. 1a). This is because the process of defocusing - by using compression optics - is equivalent to translating the ILIDS image plane along the axis of the imaging lens, while keeping the ILIDS array parallel to the PIV array. Though translation of the image plane does not allow image compression, it is assumed that, in the ILIDS plane, the defocused image is compressed in $y$ direction (i.e. normal to the plane of the paper) and defocusing is done only in the $z$ direction (Fig. 2b). 


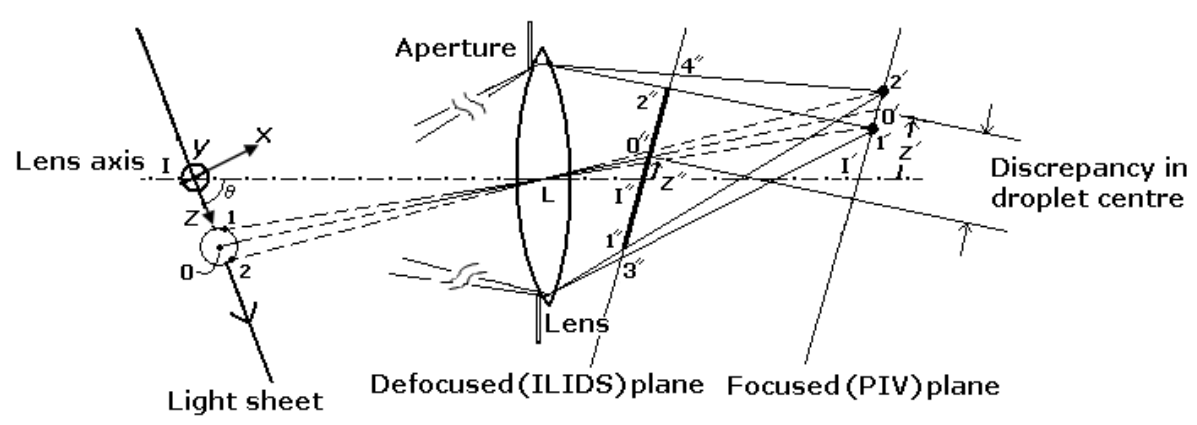

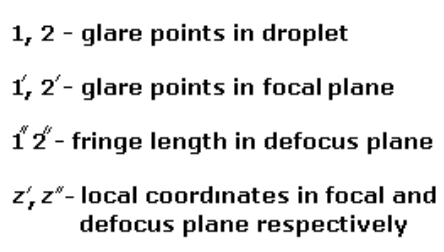

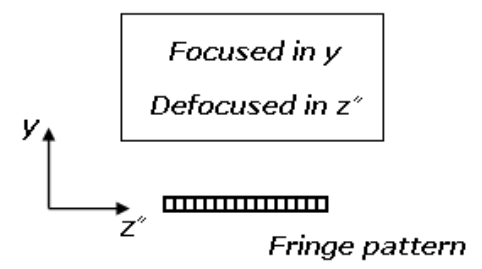

Defocus (ILIDS) plane
$\mathbf{O}^{\prime}$ - centre given by projection of point $\mathbf{0}$ in the focal plane

$0^{\prime \prime}$ - location given by intersection of line $00^{\prime}$ with defocus plane

$1^{\prime \prime} 3^{\prime \prime}, 4^{\prime \prime}-$ non overlapping length

$I^{\prime \prime} I^{\prime} / L I^{\prime}$ - degree of defocus

(a)

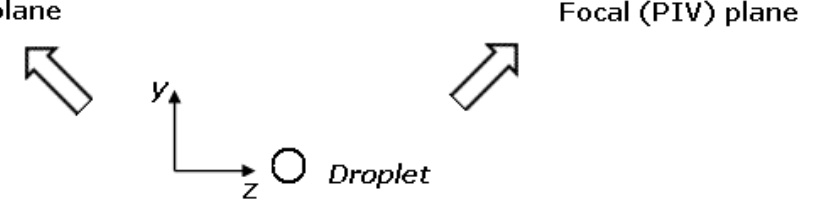

Object plane (Laser sheet)

(b)

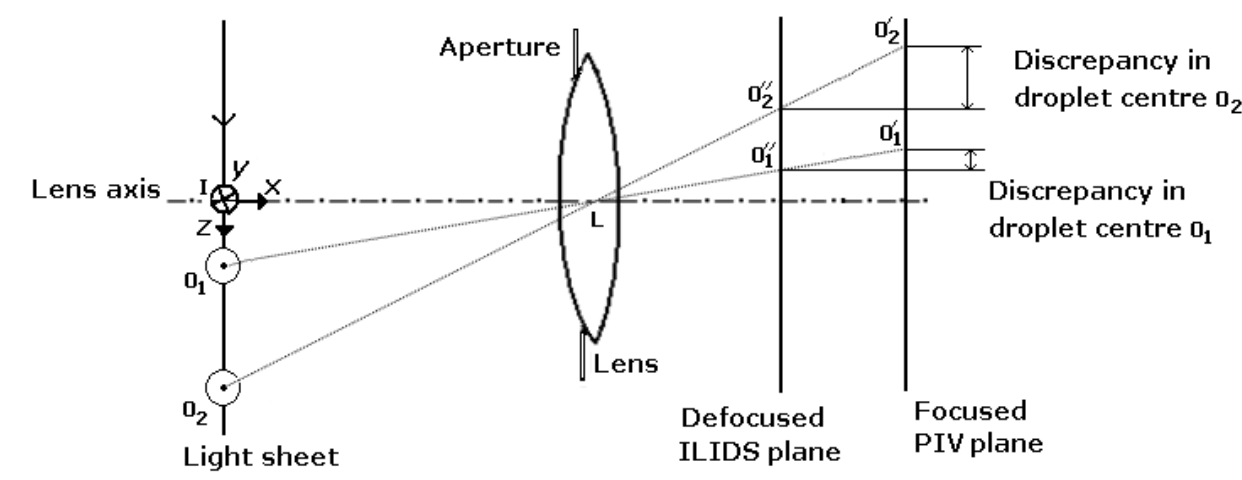

(c)

Fig. 2 (a) Schematic of the optical system for theoretical prediction of discrepancy in droplet centres between the focused and defocused planes. (b) Images along the axis of the lens of a droplet in the focussed and defocused planes and the corresponding coordinate systems. (c) A simplified schematic of the same optical system shown in Fig 2a (but with the laser sheet, focused plane and defocused plane normal to the lens axis) in order to depict the dependency of the centre discrepancy on the droplet position. 
Figure 2a shows a droplet of diameter $D$ situated at a distance $z$ from the point where the lens axis intersects the laser sheet. The position of the glare points 1 and 2, formed by reflection and refraction at the droplet surface, with respect to the centre can be calculated from a geometrical optics light scattering model (Golombokyz et al. 1998). The CCD imaging plane for PIV is inclined to satisfy the Scheimpflug condition. In this in-focus (PIV) plane, the glare points are imaged to points $1^{\prime}$ and $2^{\prime}$ with the centre of the droplet imaged to $0^{\prime}$ which we call the 'derived centre': the geometrical centre of the image, in contrast, is mid-way between $1^{\prime}$ and $2^{\prime}$. The calibration of the ILIDS and the PIV CCD arrays is carried out with the ILIDS optics adjusted to be in focus so the coordinate systems fixed to the focussed ILIDS and PIV CCD arrays, $z^{\prime \prime}$ and $z^{\prime}$ respectively, both have their origin on the axis of the lens. After calibration, the ILIDS image is defocused by shifting the image plane towards the lens, the degree of defocus ${ }^{2}$ being the ratio of the distance between the centres of defocus plane to in-focus plane $\left(I^{\prime \prime} I^{\prime}\right)$ to that of centres of lens to in-focus plane $L I^{\prime}$. The geometrical centre of the ILIDS image is the mid-point of the fringe pattern $1^{\prime \prime} 2^{\prime \prime}$. The point of intersection of the ray $00^{\prime}$ with the defocused ILIDS plane is $0^{\prime \prime}$ (which is generally not coincident with the geometric centre of the ILIDS fringe pattern) and is the "derived centre" of the fringe pattern. Figure $2 \mathrm{a}$ shows that location 0 " is offset, in $z^{\prime \prime}$ coordinates, from $0^{\prime}$.

We define the terminologies as follows: 'centre deviation' is the difference in location of the geometric centre from the derived centres of a given droplet's image, in either the in-focus or the defocus planes, and is a signed quantity. Similarly, the 'centre discrepancy' is the difference in either the derived or the geometric centres between in-focus and defocus plane and is also a signed quantity. The centre deviation and discrepancy are qualitatively demonstrated by the schematics inset to Fig. 3a and $3 \mathrm{~b}$ respectively. Unless otherwise mentioned, the term 'centre discrepancy' will refer to the geometrical centres of the fringe pattern and glare points - the geometrical centres being the only experimentally accessible aspect of the above images.

The input parameters for the analysis, as for the experiment, were: $\theta=69^{\circ}, D=0.2 \mathrm{~mm}$, object distance $=360 \mathrm{~mm}$, focal length of the lens $=135 \mathrm{~mm}$, image distance $=216 \mathrm{~mm}$ and aperture size $=$

\footnotetext{
${ }^{2}$ Note that quantifying the degree of defocus in a given experiment is not straight forward since it is difficult to determine the distances $I^{\prime \prime} I^{\prime}$ and $L I^{\prime}$ exactly.
} 
$40 \mathrm{~mm}$. In order to satisfy the condition of far-field optics for ILIDS, the defocus distance $\left(I^{\prime \prime} I^{\prime}\right)$ was chosen to be $40 \mathrm{~mm}^{3}$ : the corresponding non-overlapping length was $0.2 \%$ of the fringe length and the degree of defocus was 0.19 . The following conclusions were derived from the analysis.

1. In the PIV plane, the "centre deviation", shown as open circles in Fig. 3a, is of the order of $0.02 \mathrm{~mm}$ - a small fraction of the droplet diameter which is almost independent of the position $(z)$ of the droplet in the object plane but decreases proportionally with droplet size. Thus the mid-point of the glare points corresponds closely to the location of the centre of the droplet, with an error of only $0.1 \mathrm{D}$.

2. In the ILIDS plane, the "centre deviation", shown as asterisks in Fig. 3a, is of the order of $0.07 \mathrm{~mm}$ and is proportional to the degree of defocus. Its magnitude is about one third of the droplet diameter and so it cannot be considered negligible. The "centre deviation" does not, however, depend strongly on either the droplet size or position in the object plane. Hence, when the experimental results from ILIDS and PIV were finally combined, the positions of the droplets were found from the centre of glare points.

3. The "centre discrepancy" between the centres of the glare points and the fringe pattern, in the in-focus and defocus planes respectively, was determined for both the derived (the distance $0^{\prime} 0^{\prime \prime}$ in Figure 2a) and geometrical centres. For both cases, the discrepancy is, as expected, proportional to the degree of defocus and dependent on the droplet's $z$ position. Figure $3 \mathrm{~b}$ shows that the centre discrepancy (for either derived or geometric centre) varies almost linearly across the image plane, from being negative on one side of the image plane to positive on the other side. For the particular parameters considered here, the maximum discrepancy ${ }^{4}$ is towards the edge of the CCD array and can be of the order of $1 \mathrm{~mm}$, or five times greater than even the relatively large droplet diameter considered here. The dependency of the centre discrepancy over the droplet position is schematically shown in Figure $2 \mathrm{c}$ for two different positions of the droplet in the object plane. To avoid complexity in the ray diagram, the laser sheet and, hence, the defocused/focused plane were kept

\footnotetext{
${ }^{3}$ Note that, this is an arbitrarily selected value and the corresponding distance in the experiment presented later is different.

${ }^{4}$ This discrepancy, here related to the use of the geometric centre, arises even if we were able to use the 'derived centre' of the glare points/fringe pattern.
} 
normal to the lens axis. Larger discrepancy for the droplet position away from the lens axis can be observed.

4. The dependence of centre discrepancy (with respect to the original position) across the thickness of the laser sheet is plotted, in Figure 3c, as a function of position $x$ (see figure 2a) for three $z$ positions of the droplet. The thickness of the laser sheet was taken as $2 \mathrm{~mm}$. The maximum change in discrepancy is about $\pm 0.04 \mathrm{~mm}$, corresponding to about a fifth of the diameter, $D$, as the droplet moves within the thickness of the laser sheet, for $x$ equal to $1 \mathrm{~mm}$ to $+1 \mathrm{~mm}$.

5. The magnitude of 'centre discrepancy' was almost independent of droplet size. For example, the difference in discrepancy between a droplet of $200 \mu \mathrm{m}$ and a $20 \mu \mathrm{m}$ diameter droplet in figure $3 \mathrm{~d}$ is only $2 \mu \mathrm{m}$. The origin of the observed small dependence of centre discrepancy on droplet size is the non-symmetric position of the glare points around the droplet centre in the image plane, which varies with droplet size.

6. Though not presented here, calculation without the Scheimpflug condition in the defocus (ILIDS) plane showed that the magnitude of the discrepancy remained almost unaltered (but, as expected, it reduced the magnitude of centre deviation in the defocused plane by a factor of 10).

The conclusions drawn above are valid for all far field defocused planes where the 'non overlapped length' outside the fringe is negligible $(\sim 1 \%)$ compared to the fringe length.
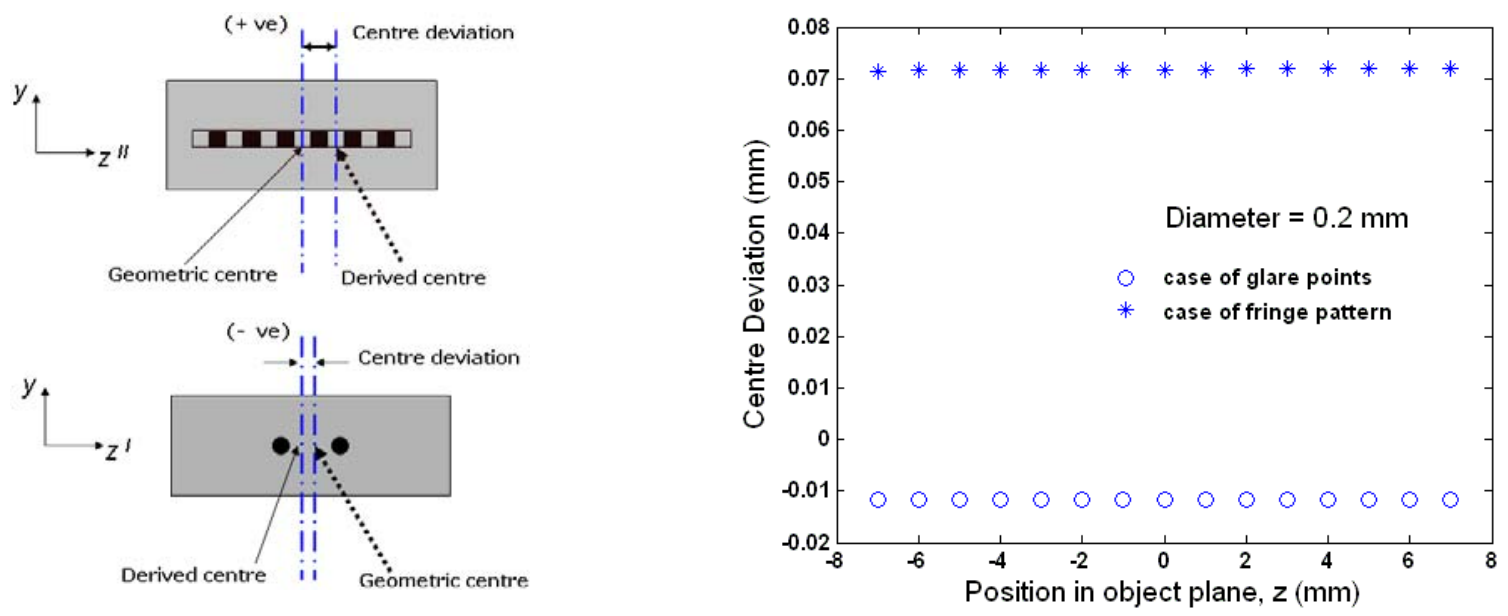

(a) 

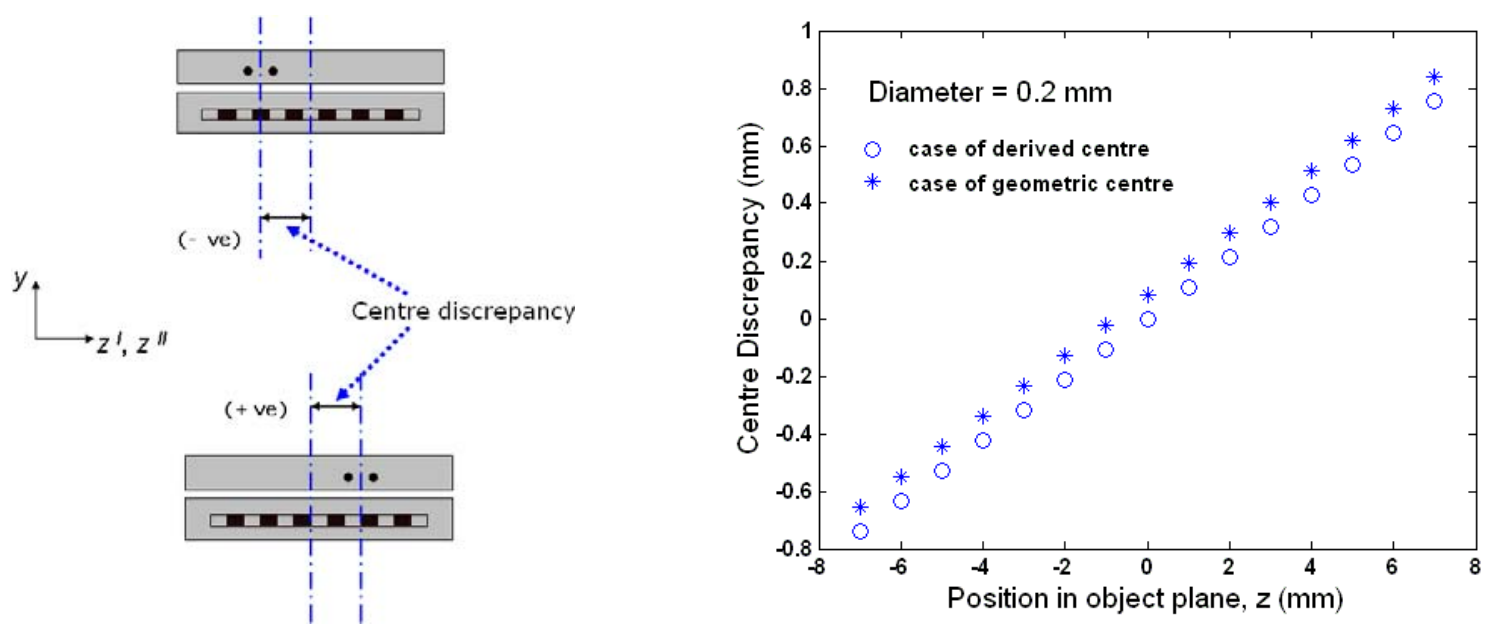

(b)

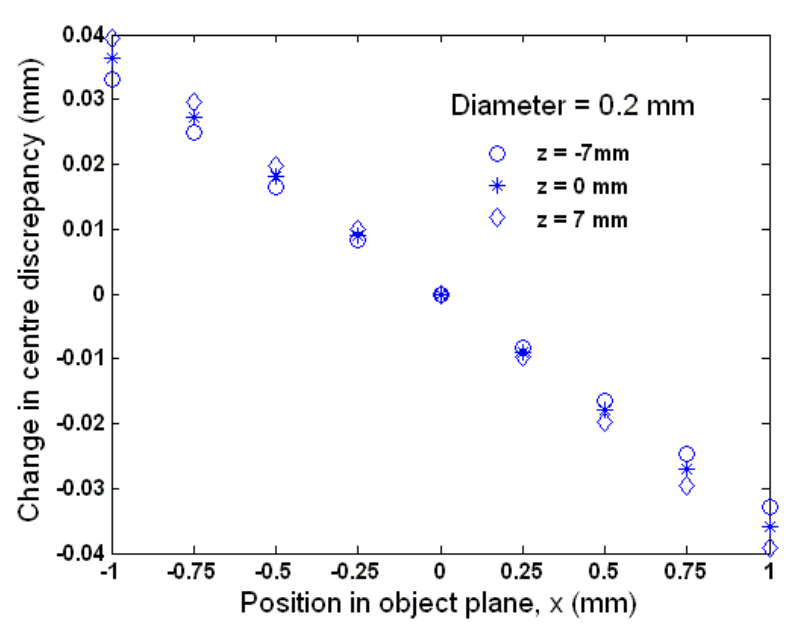

(c)

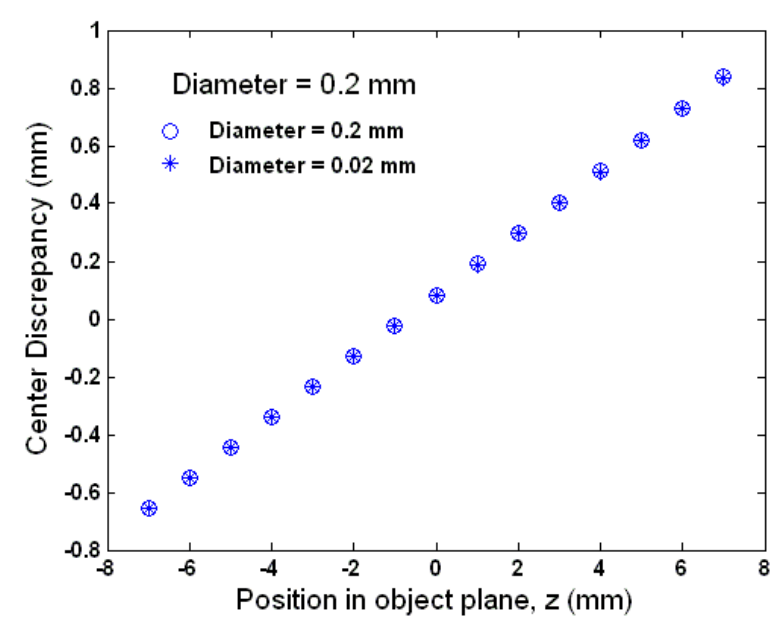

(d)

Fig. 3 (a) Deviation of the geometric centre from the derived centre of the droplet image as a function of $z$ position in the object plane. (b) Discrepancy in derived centre and geometrical centre between in-focus and defocus planes as a function of $z$ position in the object plane. (c) Relative change in centre discrepancy as a function of position $x$ in the object plane for a droplet situated at three positions $z=-7,0$ and $7 \mathrm{~mm}$ respectively. (d) Comparison of the centre discrepancy for two droplet sizes of $200 \mu \mathrm{m}$ and $20 \mu \mathrm{m}$ as a function of position $z$. Focal length was $135 \mathrm{~mm}$, Magnification was 0.6 and Aperture size was $40 \mathrm{~mm}$.

The theoretical results presented above are limited by the simplification in the calculations used to avoid making the analysis cumbersome. Also the theory does not take into account the position of the droplets in $y$ direction (perpendicular to the plane of paper). Hence, in order to quantify the droplet centre discrepancy in a practical arrangement, particularly under the defocus setting of the compression optics in the ILIDS arrangement, we rely on experiments. 


\section{Quantification of droplet centre discrepancy}

\subsection{Detection of droplet centre}

Figure 4 shows the simultaneous defocused and focused images obtained with the optical set-up described in section 2 from a stream of water droplets issuing from the monodispersed droplet generator without co-flowing air. Each droplet can be observed in Figure 4 as a set of horizontal stripes of light in the ILIDS image (left; vertical fringes exist but cannot be seen at the resolution of the camera) and as a pair of glare points in the PIV image (right).
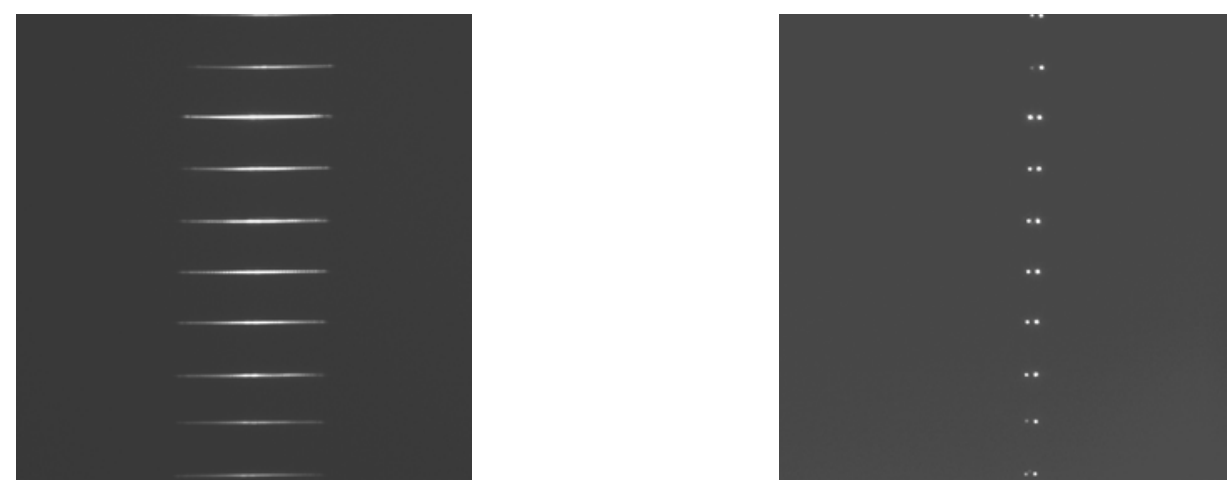

Fig. 4 Simultaneous defocused (left) and focused (right) images of droplets (diameter $228 \mu \mathrm{m}$ ) generated by a monodisperse droplet generator (droplets flow downward).

Since identification of the location of droplet centres plays a vital role in subsequent image processing, the first step is to describe the method of detection of the fringe patterns in the ILIDS image, and of the glare points in PIV images. The algorithm employed in this work used the Continuous Wavelet Transform (CWT), as explained by Sugimoto et al. (2006): its advantage is that, unlike a Fourier transform, the wavelet transform can provide frequency information which is localised over the CCD array. The transformation is obtained as the convolution of the discrete signal with the scaled and translated version of the mother wavelet (Torrence and Compo, 1998). The peak values of the wavelet transform spectrum indicate the position in the image where the particular frequency (corresponding to the scale of the wavelet) exists. For the processing of ILIDS images, the CWT was applied along each horizontal line of the image for two different scales of the mother wavelet or wavelet basis (the Mexican Hat wavelet was chosen for this purpose due to its strong localisation 
properties). Figure 5a shows a typical fringe pattern and the corresponding wavelet transforms at different scales. The transformation at larger scale (approximately equal to the fringe length) resulted in a wavelet transform spectrum with maximum almost at the geometric centre of each fringe pattern, while the transformation at the smallest scale yielded maxima corresponding to each intensity modulation of the individual fringes. The fringe pattern length was found by considering the approximate centre identified by the large scale transform and maxima yielded from the transformation at smaller scale and by using a suitable, but arbitrary, intensity threshold based on the mean intensity of the fringe. Thus the 'droplet centre' is located at the geometrical centre of the fringe pattern. The droplet size was measured by applying the Fast Fourier Transform along a horizontal line of the fringe pattern to identify the fringe spacing from the power spectrum. Particle Tracking Velocimetry between two images, obtained at consecutive times, quantified the droplet velocity from the corresponding droplet displacement. The precision in locating the centre of the fringe patterns in the ILIDS image, for the case of spray, was estimated as being \pm 20 pixels in horizontal direction and \pm 1 pixels in vertical direction. In the case of the droplet generator, the precision in centre location was better $( \pm 6$ and \pm 1 pixels in horizontal and vertical directions respectively) because of higher signal to noise ratio of the fringe patterns since multiple scattering was absent, in contrast to spray.
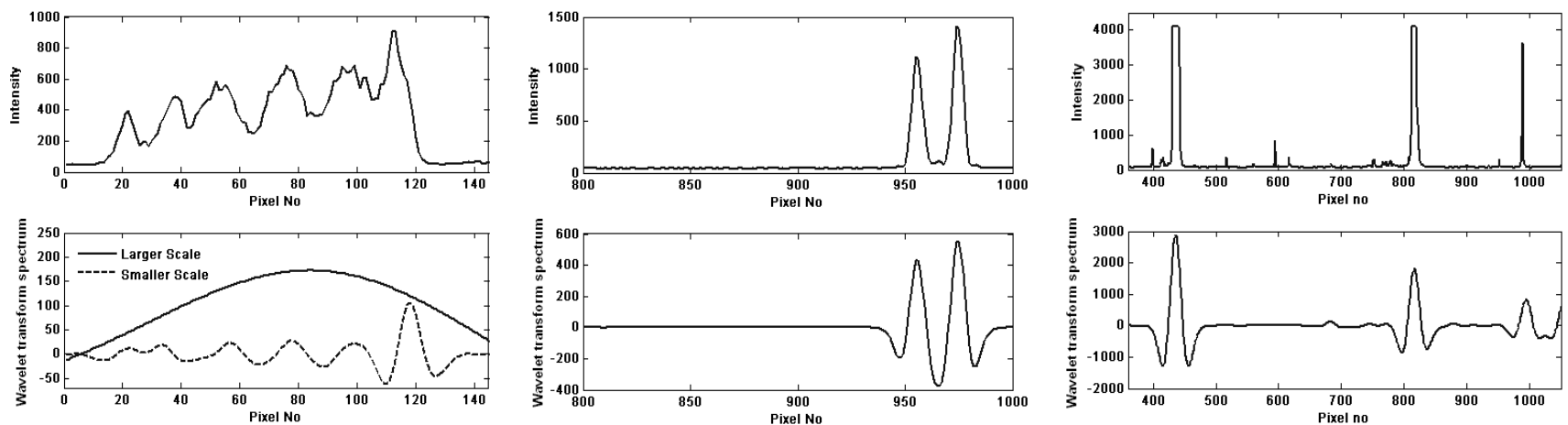

(a)

(b)

(c)

Fig. 5 (a) A typical intensity variation for a fringe pattern in the ILIDS image of a droplet and its corresponding Continuous Wavelet Transform spectrum at two different scales (left), (b) Intensity distribution of the Glare points in the PIV image corresponding to the droplets of Fig 4 and its corresponding wavelet transform spectrum and (c) Intensity distribution and wavelet transform spectrum of the glare points corresponding to the polydisperse spray.

The glare points of each droplet in the PIV image were detected, again, by applying a wavelet transform with a small scale along each line of the image using the same mother wavelet as before. As 
shown in Fig 5b, each maximum obtained from the wavelet transform spectrum corresponds to the location of a glare point. The geometrical centre between the two adjacent maxima (glare points) was assumed to be the actual centre of the droplet. From geometrical optics, it can be shown that for each droplet, the distance between the two glare points is proportional to the droplet size (the droplet diameter is about 1.4 times this distance) and thus can be a measure of droplet diameter. However, the determination of droplet diameter through this approach is not reliable. This is because it is not always possible to resolve the distance between the glare point pairs, even for relatively large droplets, due to restrictions in CCD array resolution. The demand of higher spatial resolution and the necessity for magnification by the receiving optics causes the method to have no distinct advantages over direct imaging (Damaschke et al. 2005 and Kawaguchi et al. 2002). Diffraction limited imaging also imposes a restriction on the minimum measurable droplet size. Finally, the theoretical model (Section 3) shows that the relative distance between the two glare points of a given droplet can vary across the CCD as the droplet changes its position in the object plane. The minimum measurable droplet diameter, through glare points, was of the order of $100 \mu \mathrm{m}$ for our experimental conditions. Hence, in this work, the glare points were used only to detect the location of droplets on the PIV image. The corresponding droplet glare points at the later time of a pair of PIV images were found through particle tracking velocimetry, similar to the approach used for ILIDS.

Note that in Figure 5b, which corresponds to the large droplets issuing from the droplet generator, the intensity profile can provide the glare point centre directly without using the CWT. However the same approach leads to ambiguity in the polydisperse spray. Shown in Figure 5c is the intensity profile and the CWT spectrum of some of the glare points identified in the PIV image of the spray with the co flowing air containing the seeding particles. Unlike Fig .5b, the two intensity maxima for each pair of glare points are no longer resolved and the intensity of the glare points also saturates the camera. So the detection of centre of glare points based only on intensity can be ambiguous. In contrast, the peak in the CWT spectrum provides a good approximation in this regard. The precision of locating the centre of glare points in PIV image was estimated as \pm 2 pixels in both horizontal and vertical directions. Use of CWT also has the additional advantage of discriminating the glare points from the seeding particles in the PIV image. By selecting an appropriate scale of the wavelet, it is possible to suppress the peaks produced by the seeding particles in the CWT spectrum and then, by selecting a suitable threshold, glare points only are identified, thereby eliminating signals from seeding particles. For example, though the peak of the intensity profile at the extreme right of Fig .5c has 
amplitude similar to the other two peaks, its corresponding CWT spectrum has lower amplitude and is eliminated as a seeding particle.

\subsection{Quantification of the centre discrepancy by droplet generator}

Once the droplets are detected in both ILIDS and PIV images, the next task is to identify the glare points in the PIV image corresponding to the appropriate fringe pattern in the ILIDS image. However, the discrepancy in identifying the location of the droplet centre between the two images reduces the probability of identifying the correct glare points, especially for the case of a spray. It is essential to quantify the magnitude of the discrepancy and eliminate it. Hence, experiments were performed with the droplet generator at five different positions along the " $z$ " dimension shown in Figure 2. The degree of defocus was kept the same for all cases by maintaining the same position of the cylindrical lenses in the optical compression unit. The corresponding images are shown in Figure 6 for three different positions only due to space restriction. In order to demonstrate the variation of droplet centre position, the focused and defocused images have been superimposed in Figure $6^{5}$. The glare points seem to be moving from left to right with respect to the fringe centre with increasing $z$ location. Two sets of images were processed for each position. At first, the locations of the droplet geometrical centres in ILIDS and PIV images were obtained by processing these separately. Then these were projected to the object plane using calibration coefficients ${ }^{6}$. A fringe pattern was associated with a pair of glare points if the difference in their resulting image centres in the object plane was minimum in both horizontal $(z)$ and vertical $(y)$ direction. Since in this case the droplet number density was low, and also there was no seeding in the air, the probability of correctly identifying the corresponding glare points with a fringe pattern was almost $100 \%$. The discrepancy between the centres was calculated for both horizontal and

\footnotetext{
${ }^{5}$ the location of the glare points and the corresponding fringe pattern in this case were misaligned in the vertical direction.

${ }^{6}$ another way of dealing with the problem of droplet centre discrepancy would be to have the ILIDS camera defocused during calibration: but then the detection of the "defocused crosses (calibration marks)" is more difficult. Also, this approach is based on an assumption that if the droplet centre and the centre of the "mark" coincide in the object plane initially, than after defocusing, the geometric centre of the "defocused mark" coincides with the geometric centre of the defocused droplet image as well. However this may or may not be true, because of the fact that the glare points are not symmetrically spaced around the droplet centre. Nevertheless the resulting error is likely to be small. Hence the approach of calibration before defocusing was preferred in this work which has the additional advantage of permitting the change of the degree of defocus, if required, during an experiment.
} 
vertical directions. The droplet diameter $(D)$ estimated by ILIDS was $228 \mu \mathrm{m}$, which is within $7.5 \%$ of the theoretically calculated value for the operation of the monodispersed droplet generator.

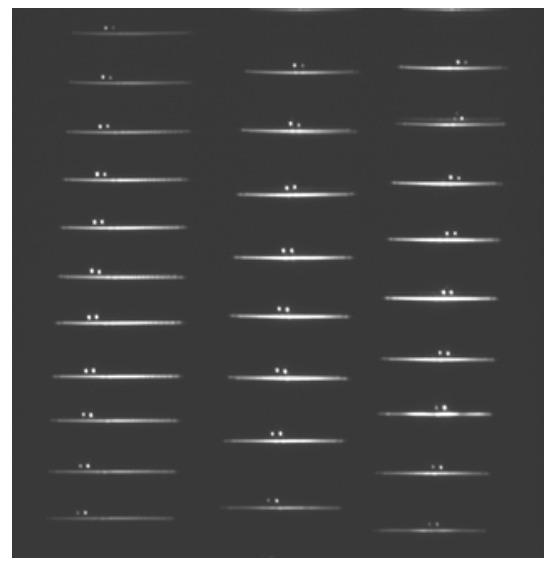

Fig. 6 Fringe patterns and corresponding glare points for a stream of monodispersed droplets without gas phase seeding at three different positions in the object plane, $z=-5.0,+1.0$ and $+5.0 \mathrm{~mm}$ respectively. The ILIDS images are superimposed on the PIV image. Note that the origin of the coordinate system $(z=0$ and $y=0)$ lies in the centre of the CCD array, which coincides with the centre of the image of the figure.

The droplet centre discrepancy as a function of horizontal, $z$, position in the object plane is shown in Figure 7a and the discrepancy can be as large as $1000 \mu \mathrm{m}$, which is about 5 times the droplet diameter in the present case. The droplet stream was found to have been inadvertently inclined (by about $5.5^{\circ}$ ) relative to the vertical $(y)$ direction of the image ( $y z$ plane) during experiment. So, for each (nominal) position in the $z$ direction, a spread in the measured error can be observed. The variation of the discrepancy with $z$ is close to linear and the magnitude is greater than the droplet size (towards the edge of the CCD array). Although it is hard to make direct comparison with the theory of section 3 (because it is difficult to quantify the defocus distance experimentally), the magnitude of the centre discrepancy (i.e. the vertical scale) in Figure 7a is comparable to that expected from the analysis, as shown in Figure 3b. The overall trend of the error can be described empirically through a linear fitting and ultimately can be virtually eliminated from the original values. The residual error is plotted in Figure $7 \mathrm{~b}$ and was less than a droplet diameter (maximum of about half of the droplet size) in this case.

This remaining error can be due, in principle, to various sources such as additional inclination relative to the vertical in the $x y$ plane, i.e. normal to the plane of the laser sheet (which leads to variation in object distance of the droplets), inaccuracy in camera calibration and the error involved in detection of fringe centre and glare point centre in ILIDS and PIV processing respectively. However, according to the theoretical analysis (Fig. 3c), the error due to out-of-plane movement of the droplets 
within the laser sheet, in our case, is only of the order of $20 \mu \mathrm{m}$. The inaccuracy in camera calibration is only of the order of $15 \mu \mathrm{m}$ and, the precision in the detection of centre of the glare points and the centre of the fringe pattern are of the order of $20 \mu \mathrm{m}$ and $100 \mu \mathrm{m}$ respectively. We conclude from comparison of the above cases that the cause of the residual error can be attributed primarily due to the inaccuracy of detection of the fringe centre. It should be noted that the experimental measurements of Figure 7 are not directly comparable to the theoretical analysis of Figure $3 \mathrm{~b}$. This is due to, as explained earlier, the unknown defocus distance of the lens in the experiment, which is different from the indicative $40 \mathrm{~mm}$ distance used in the theoretical section. As a consequence, the location of the minimum centre discrepancy occurs at different location along the CCD array and, is not the same as indicated in Figure $3 b$.

In the vertical (y) direction, the trend of the discrepancy (Fig. 7c) was found to slightly increase from the top to the bottom of the CCD array with magnitude between $100 \mu \mathrm{m}$ and $200 \mu \mathrm{m}$, which is much smaller than the error obtained in the $z$ direction and can be considered to be constant. Since no defocusing was performed in the $y$ direction, ideally the discrepancy should be zero in this case. Thus its appearance can be thought of being related to the systematic error in the calibration of the optical arrangement with both cameras and also to the inaccuracy in droplet centre location during image processing. Similarly to the case of the discrepancy in the $z$ direction, the error was represented through a linear fit and subtracted from the original values, which resulted in a final remaining error of the order of $40 \mu \mathrm{m}$.

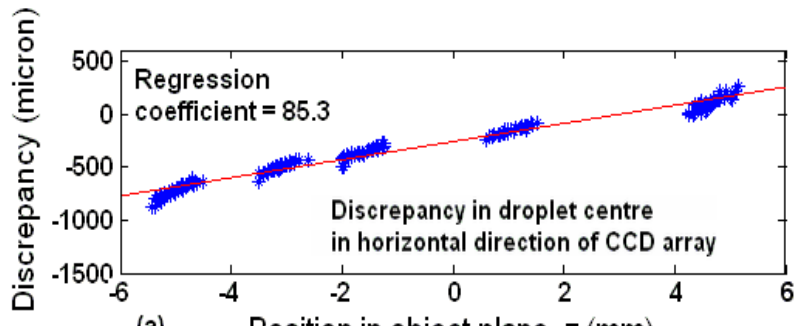

(a) Position in object plane, $z(\mathrm{~mm})$

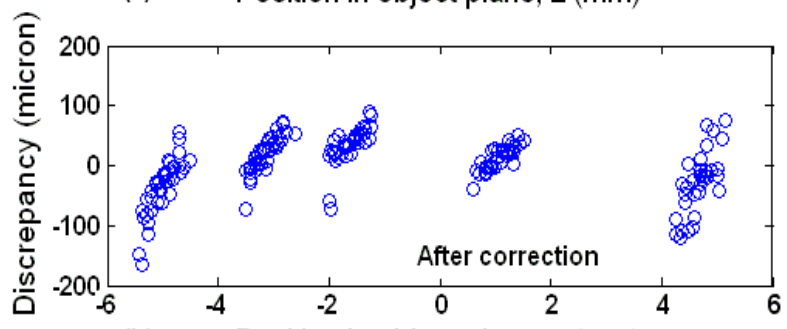

(b) Position in object plane, $z(\mathrm{~mm})$

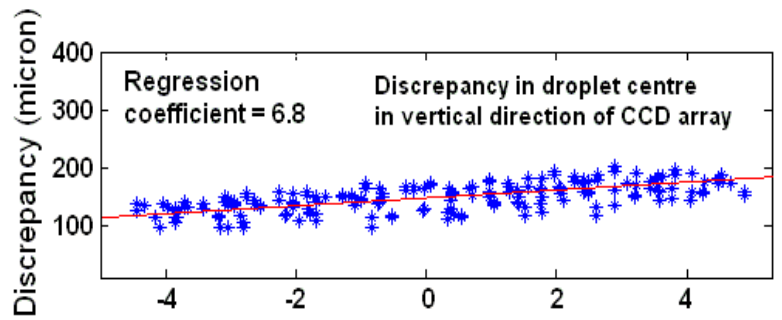

(c) Position in object plane, y $(\mathrm{mm})$

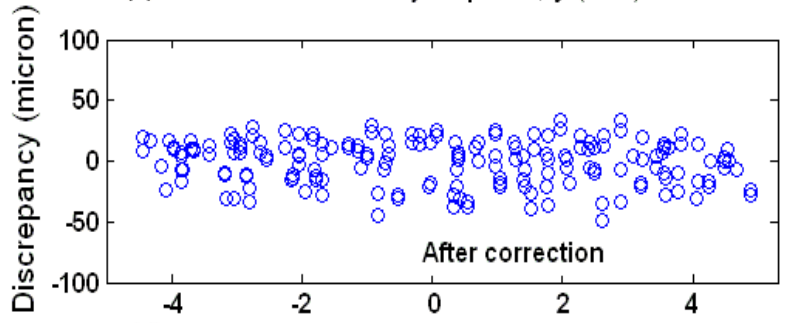

(d) Position in object plane, y $(\mathrm{mm})$ 
Fig. 7 (a) Centre discrepancy between ILIDS and PIV images in the horizontal ( $z$ ) direction as a function of $z$ position in the object plane. The straight line is a linear fit to the original data. (b) The remaining droplet centre discrepancy after subtracting the estimated error of Fig. 7a. (c) Centre discrepancy in the vertical ( $y$ ) direction as a function of $y$ position in the object plane and the estimated error. The straight line is a linear fit to the original data. (d) The remaining droplet centre discrepancy after subtracting the estimated error of Fig. 7c

\subsection{Application to a polydisperse spray with no gas phase seeding}

The matching of a fringe pattern with a pair of corresponding glare points becomes more challenging for a spray, relative to the case of monodispersed droplets, because the droplet number density is much higher and because the inter-droplet distance is random. The measurements in a spray were performed for a relatively dilute region at $50 \mathrm{~cm}$ below the nozzle centre and $20 \mathrm{~cm}$ away from the nozzle axis. The injection pressure was 1 bar and volume flow rate of water was $10.83 \times 10^{-4} \mathrm{~kg} / \mathrm{s}$. The Sauter Mean Diameter (SMD) was $68 \mu \mathrm{m}$ as estimated from ILIDS. The droplet number density was about 25 droplets/image as compared to 10 droplets/image for the case of the droplet generator. The corresponding ILIDS and PIV images are shown in Figure 8. In total 10 pairs of image pairs were processed to obtain droplet centres. In the focused image, detection of the pairs of glare points was more difficult (as compared to the case of the droplet generator with much larger droplet size) because these tended to overlap for the smaller droplet sizes and could appear as a single bright spot instead of two. In such cases only one peak was obtained in the wavelet transform (refer to Fig. 5b), the position of which was then assumed to be the droplet centre. According to the analysis, the discrepancy between droplet centres from focused to defocused images is almost independent of droplet size. Therefore, the estimated error in centre discrepancy in both $y$ and $z$ directions, obtained for the monodispersed droplets (by linear fitting of the measured discrepancies), should be the same for a spray with a distribution of droplet sizes, since the degree of defocus of the ILIDS optics was not changed. The respective estimated errors, depending on the $z$ position in the object plane, were subtracted from the position of the centre of each fringe pattern in the ILIDS images. For each of the fringe patterns, the corresponding pair of glare points was sought within a search window, defined in the object plane. The size of the search window was set by the remaining discrepancy in both directions obtained in the previous section (Fig. $7 \mathrm{~b}$ and $7 \mathrm{~d}$ ) using the monodispersed droplet stream. In the present case it was set to $100 \mu \mathrm{m}$ in both directions. A smaller size of search window would result in higher accuracy in finding correct pairs of glare points and fringe pattern but could simultaneously decrease the probable 
validated pairs and hence a compromise had to be made. If more than one candidate was present, the one with minimum error in both $z$ and $y$ direction was chosen.
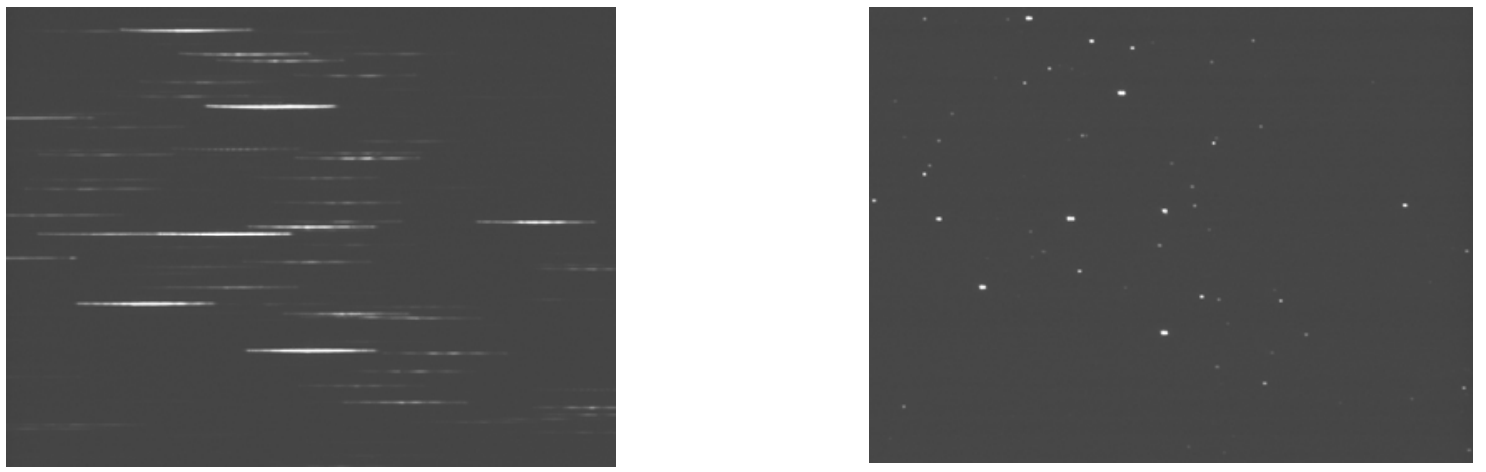

Fig. 8 Simultaneous ILIDS (left) and PIV (right) images of droplets in a polydisperse spray without seeding particles

In total, 68 droplets were detected on the ILIDS images, of which 50 had their corresponding glare points identified immediately. Since the defocusing inevitably caused a small increase in the viewing area with respect to the focused image due to the way in which defocusing was performed in our case, there is a possibility that the droplets near the edges of the defocused ILIDS images did not appear in the corresponding PIV images. In such cases a fringe pattern in the ILIDS image may not have any corresponding glare points on the PIV image. The final discrepancies of the droplet centres in $z$ and $y$ directions are plotted in Figures $9 \mathrm{a}$ and $9 \mathrm{c}$ respectively. In order to demonstrate the advantage of the present approach, the corresponding discrepancy without correction is also calculated and shown in Figures $9 \mathrm{~b}$ and $9 \mathrm{~d}$. The droplet centre discrepancy along the $z$ direction, with correction, reduced from about $1000 \mu \mathrm{m}$ to less than $100 \mu \mathrm{m}$. In terms of pixels this will be a reduction from about 100 pixels to below 10 pixels. Considering the SMD, the final discrepancy now becomes of the order of a droplet diameter. Similarly, in the $y$ direction, the error reduced to the same order which otherwise would have been about $200 \mu \mathrm{m}$ without correction. Without applying the correction to the location of the droplet centre, it is not possible to find the correct pairs of fringe pattern and glare point. Figures $10 \mathrm{a}$ and $10 \mathrm{~d}$ show the variation of the residual discrepancies with respect to droplet size, in both $z$ and $y$ directions respectively, which can be observed to be completely random in both cases. This is because the discrepancy has negligible correlation with droplet size, as shown in Figure 3d.

It should be mentioned here that the average inter droplet distance, for the above case, was of the order of 50 times the SMD. Thus, it is much larger than the dimensions of the search window, 
which was about twice the SMD. For a dense spray, if the inter-droplet distance reduces to about the size of search window, then the task of finding the corresponding pair of fringe pattern and glare points can become inaccurate. Hence, the present approach becomes increasingly reliable in identifying matching fringe patterns and pairs of glare points as the spray density reduces.

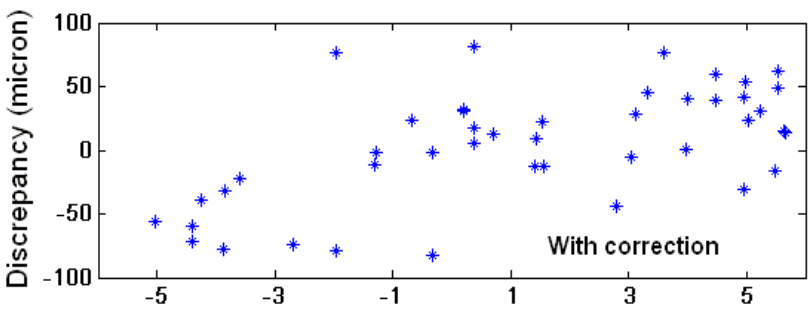

(a)

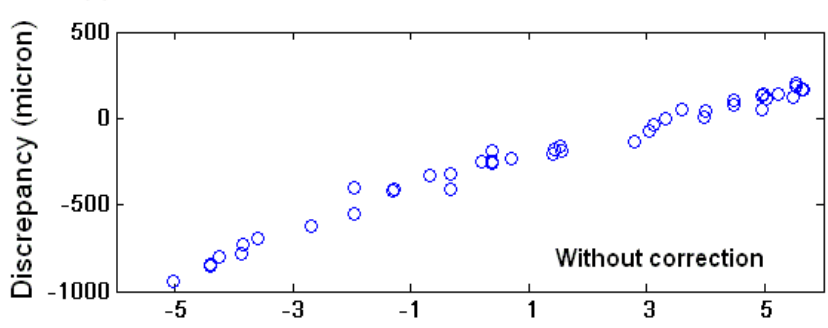

(b)

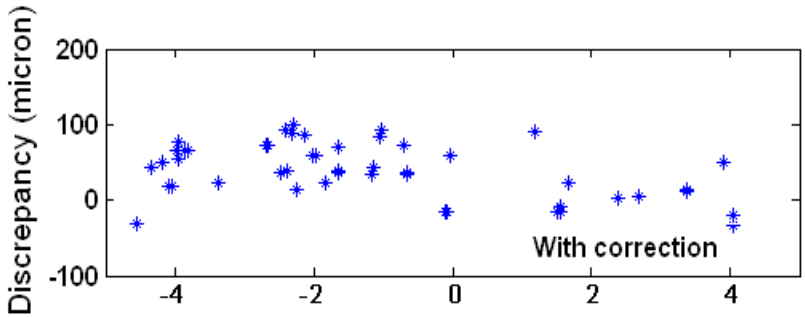

(c) Position in object plane, y $(\mathrm{mm})$

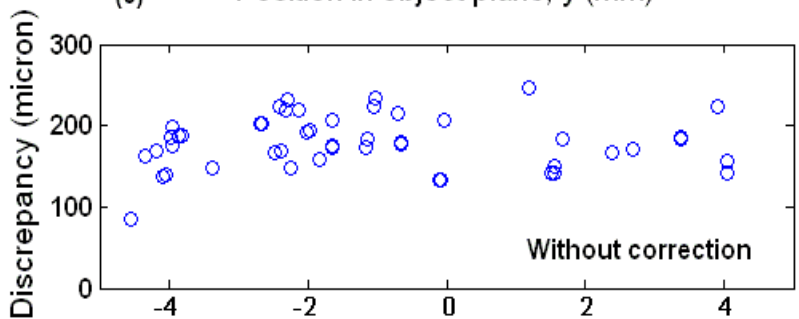

(d) Position in object plane, y $(\mathrm{mm})$

Fig. 9 (a) Droplet centre discrepancy in horizontal direction $(z)$ as a function of $z$ position in the object plane for the spray. The discrepancy was obtained after subtracting the estimated error (resulting from linear fit of the measured discrepancy for the monodisperse droplet stream) from droplet centres in the ILIDS image. (b) The same information as Fig. 9a without correction. (c) Droplet centre discrepancy in the vertical direction (y) as a function of $y$ position in the object plane. The discrepancy was obtained after subtracting the estimated error from droplet centres in the ILIDS image (d) The same information as Fig. 9c without correction

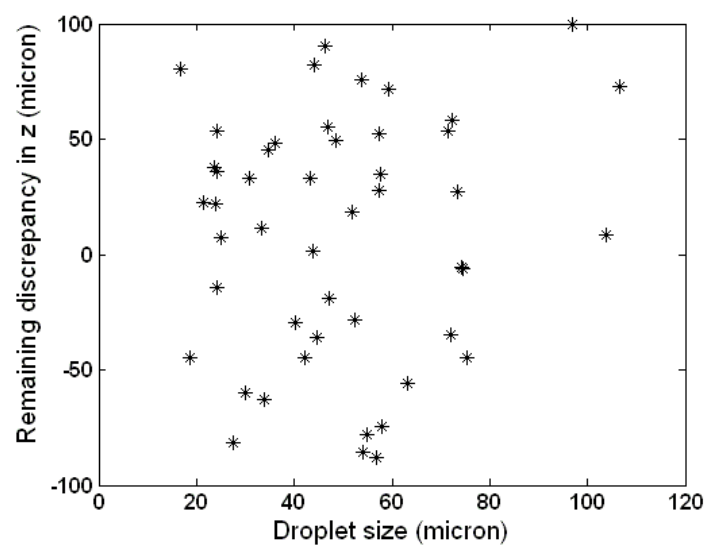

(a)

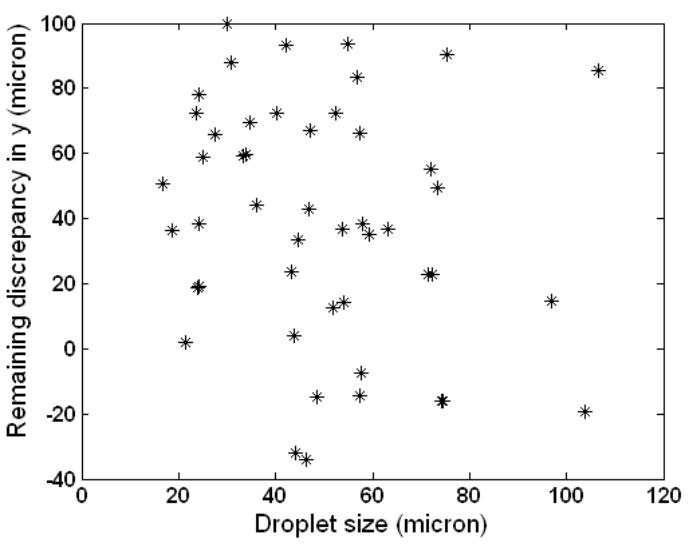

(b) 
Fig. 10 (a) Remaining droplet centre discrepancy in horizontal direction ( $z$ ) as a function of droplet size. (b) Remaining droplet centre discrepancy in vertical direction $(y)$ as a function of droplet size

\section{Combined ILIDS and PIV measurements}

In order to measure the gas velocity in the vicinity of droplets by PIV, air, seeded with aluminium oxide particles, was allowed to flow around the monodispersed droplet stream. The seeding particles were added to the co flowing air before it entered the spray rig. In this section the data processing algorithm and the corresponding results are presented for one set of experimental images obtained from the combined measurements. The same operating condition of the droplet generator was maintained as in the absence of seeding. Sequential images were collected with a time interval of $40 \mu$ s. Both 'seeding' particles and droplets were contained in the focused PIV images, in which the glare points had to be identified and removed so that the resulting images would be processed to obtain the gas velocity. Figure 11 shows simultaneous ILIDS and PIV images for the case of a monodispersed droplet stream. The overall effect of the presence of 'seeding' particles was to decrease the signal to noise ratio, making the detection of droplets more difficult. In the ILIDS image, the 'seeding' particles were also defocused and so the image looked similar to that of a dense spray. In PIV processing, the scattering intensity from a 'seeding' particle sometimes exceeded the intensity threshold imposed by the algorithm to discriminate between seeding particles and droplets. But the defocused seeding particles did not produce regular fringe patterns and so were not detected while processing the ILIDS images.
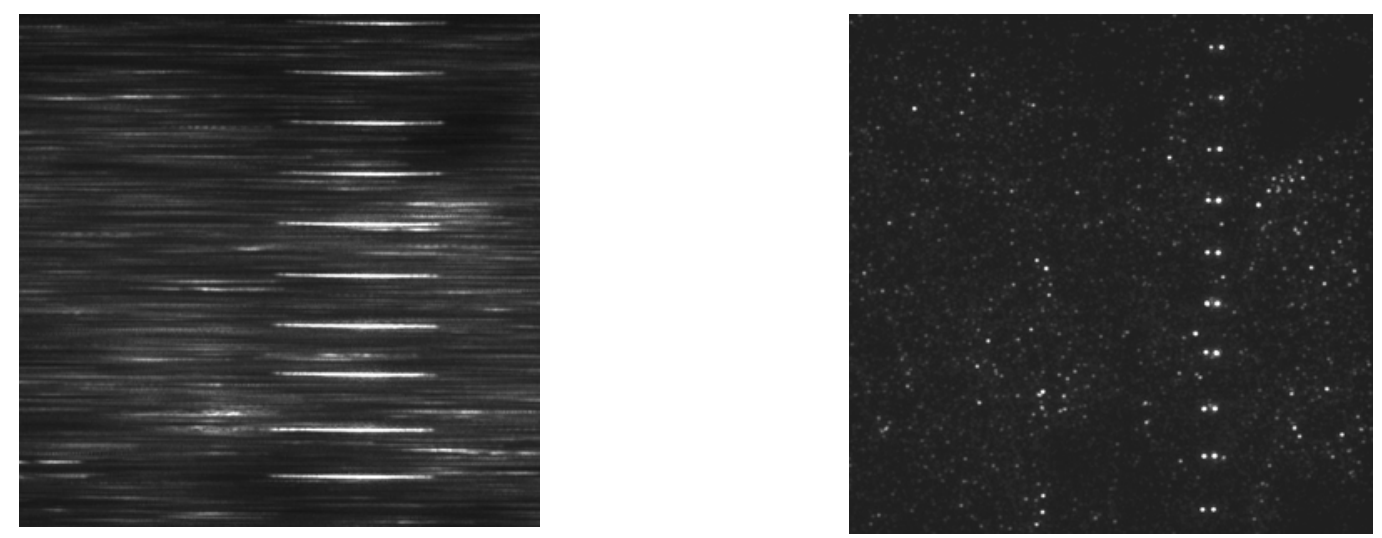

Fig. 11 Simultaneous ILIDS (left) and PIV (right) images corresponding to the monodispersed droplet stream surrounded by co flowing air seeded with aluminium oxide particles 


\subsection{Droplet filtering from PIV images}

The pair of PIV images was processed to obtain the geometric centres of the glare points and the same glare points at both time instants. The pair of ILIDS images was processed to obtain the geometric centres of the fringe patterns and the size and velocity of the droplets. For each fringe pattern, corresponding glare points were detected in the PIV images, as described in the previous section, after incorporating the correction for droplet centre discrepancy. The glare points in the PIV images were removed in order to be able to obtain gas velocity. Thus, in the PIV image, around each centre determined by the glare points, a circular area corresponding to the measured droplet diameter (from ILIDS) was removed. However, this approach has a limitation in the sense that it was not always possible to match all of the glare points in the PIV image with their corresponding fringe patterns in the ILIDS image. Also, the filtering of the glare points depends on the accuracy of diameter determination during processing of ILIDS image. Figure 12a shows the method for the removal of glare points followed in this work based on the wavelet transform. As shown in the figure, each pair of glare points resulted in three negative peaks, with the outer ones completely encompassing the positive pair of peaks. A circular region centred on the middle of the glare points, and with diameter equal to the distance between the two extreme negative peaks, can be thought of as being occupied by the droplet. Hence the pixel intensity values corresponding to this region were set to zero intensity. For overlapping glare points, the transform resulted in two negative peaks only, instead of three, and pixel intensity values were set to zero based on those peaks only.

The glare points that were removed from the PIV image were those that had corresponding fringe patterns in the ILIDS image. But it remains possible that some of the fringe patterns remain undetected in the ILIDS image, particularly when the droplet number density is too high or the signal to noise ratio is poor. This may eventually result in the corresponding glare points remaining undetected in the PIV image as well. In such cases the undetected glare points (especially from relatively larger droplets) could produce bias in the PIV correlation. Hence the algorithm also employed validation to remove any remaining glare points in the PIV image on the basis of an amplitude threshold based on the minimum intensity of successfully identified, and removed, glare points. Though such glare points could be removed in this way from the PIV image, there was no matching - or, at least, none detected fringe patterns in the ILIDS image and thus the droplet size/velocity information corresponding to those droplets was not available. The percentage of such droplets was found to be about 10 to $20 \%$ of 
the total number of detected glare points for the cases considered here. Figure $12 \mathrm{~b}$ shows a PIV image after removal of glare points.

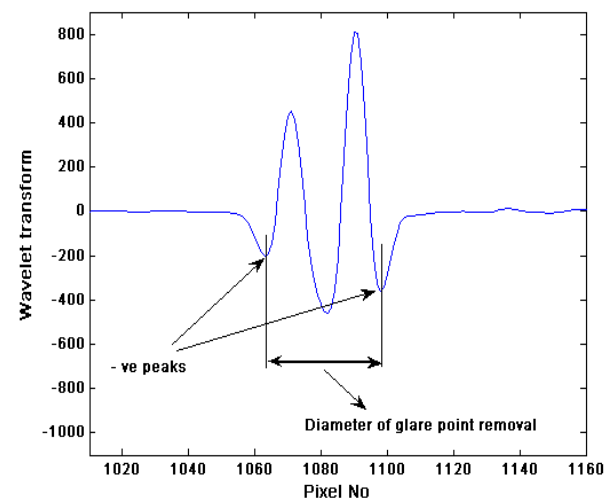

(a)

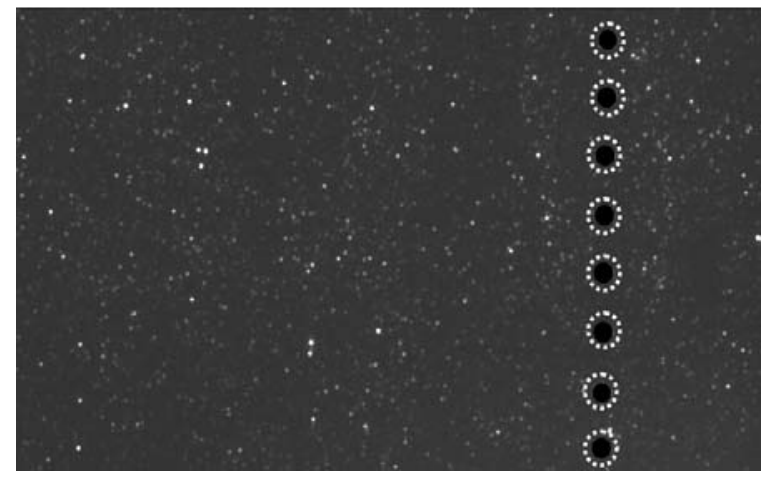

(b)

Fig. 12(a) Demonstration of identification of glare points in PIV image using the wavelet transform. (b) The PIV image after removal of the glare points (the boundaries associated with the removed glare points are shown as dotted circles)

\subsection{Calculation of the gas velocity through PIV and integration of the result with ILIDS}

The focused PIV images, filtered to retain only seeding particles, were now processed to obtain gas velocity. Use of a conventional PIV algorithm for this purpose is not advisable, because of the resulting non-uniform particle concentration due to droplet removal. Poelma et al. (2006) showed that the use of overlapping interrogation regions in images with a low image density could lead to biased results due to over sampling. This work uses an algorithm similar to that reported by Lindken and Merzkirch (2002), in conjunction with non-overlapping windows. A digital mask was created with pixel intensity values of 'zero' for the pixels corresponding to the circular region from where the glare points had been removed. A pixel intensity value of 'one' was assigned for the remaining pixels, which corresponded to the gas phase. This array was combined with the PIV algorithm during processing (Lindken and Merzkirch, 2002). In order to ensure accuracy in velocity determination, an integer based algorithm was employed instead of FFT-based processing.

It was found that when the size of the removed area in the PIV image became similar to that of the interrogation window, the error in the resulting displacement (obtained through PIV correlation) increased. This error was even greater when the displacement was of the order of one pixel or less. Since the maximum diameter of the area of the removed glare points was about 20 pixels in case of the spray and about 30 pixels in case of droplet generator, the interrogation window area was hence chosen 
to be $32 \times 32$ pixel $^{2}$ and $64 \times 64$ pixel $^{2}$ for the two cases respectively. Thus there is a trade off between accuracy in velocity and spatial resolution. The seeding density was about 5 and 10 particles within the interrogation windows in the case of the spray and droplet generator respectively. In the case of the droplet generator considered here, the seeding density could have been made higher since the larger droplet size (relative to spray) resulted in a large contrast in light intensity between the droplets and seeding particles. But, for the spray, a higher seeding density was not used as this would have adversely affected the detection of droplets and so would have resulted in a loss of validated pairs of fringe pattern and glare points.

After calculating the gas velocity, the result was integrated with that of ILIDS. This is necessary in order to assign the droplet velocity and size at the appropriate locations of the PIV images, which correspond to the location of the removed glare points. Though droplet velocity can be obtained both through ILIDS and PIV processing, the former includes diameter validation for the corresponding droplet at the two subsequent time instances. Since this is not always possible for the case of glare points in PIV, ILIDS processing is likely to be the more reliable method. However, there is one additional reason behind finding the corresponding glare points in the images at both time instances through particle tracking. Droplet size and velocity from ILIDS are assigned only to those glare points which have corresponding fringe patterns in the images at both time instances. It should be noted here that the position of a droplet in the object plane could be obtained either from its 'fringe pattern centre' in ILIDS or 'glare point centre' in PIV. However, as shown in Figure 3a, the centre deviation in the case of glare points is much smaller than in case of fringe patterns. Thus the centre of a given droplet in the object plane was decided based on the centre of the glare points. The flow chart, presented in Figure 13, summarises the logic used for the measurement of simultaneous droplet size and both droplet and gas velocity with the combined ILIDS and PIV technique.

Figure 14 shows the simultaneous velocity vectors for gas and droplets at a given instant. As expected, the gas velocity is almost negligible away from the droplet stream but increases gradually close to it. The average droplet size estimated from ILIDS was $228 \mu \mathrm{m}$. The velocity of the droplets was of the order of $10 \mathrm{~m} / \mathrm{s}$ while that of gas in the vicinity of droplets was of the order of $1 \mathrm{~m} / \mathrm{s}$. The minimum distance between the droplet centres and gas velocity vectors was about $0.5 \mathrm{~mm}$, which is expected because of the spatial resolution $(\sim 0.6 \mathrm{~mm})$, obtained from the gas velocity in the PIV image. 


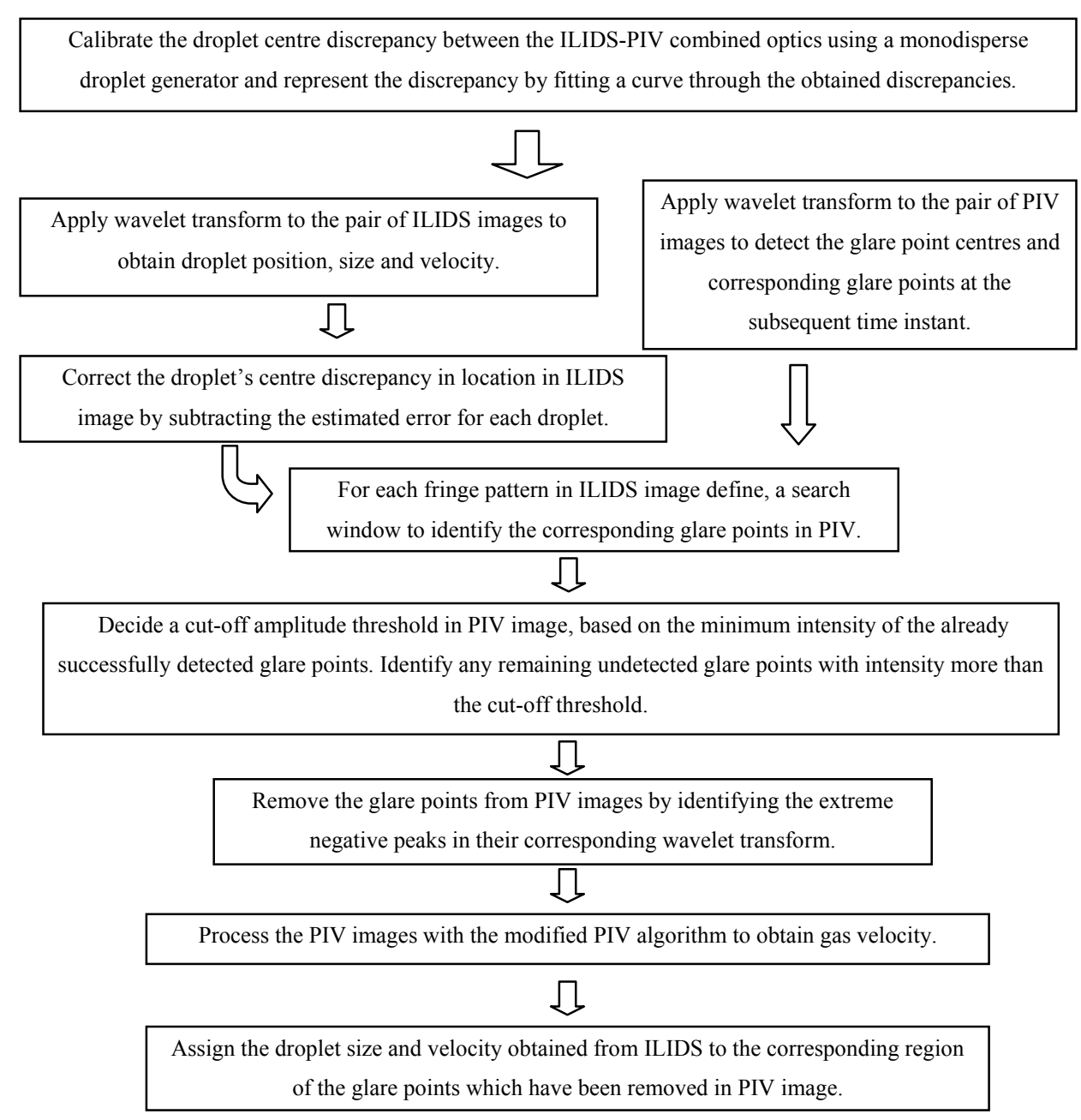

Fig. 13 Flow chart for measurements of instantaneous droplet size and both droplet and gas velocity from simultaneously recorded individual pairs of ILIDS and PIV images

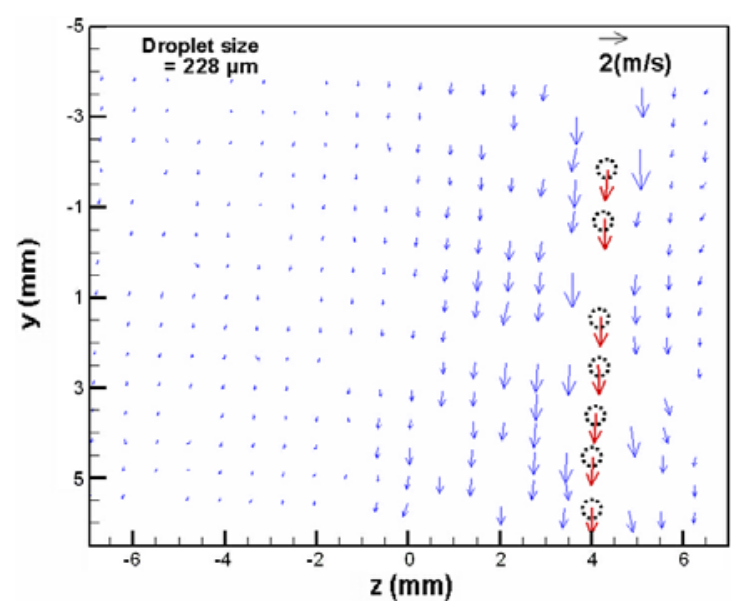

Fig. 14 Simultaneous droplet and gas velocity vector plots for the case of the monosized droplet stream surrounded with seeding particles at a given instant. Bold vectors represent droplet velocity which is scaled down by a factor of 5 
Application of the combined technique to a polydisperse spray (with seeded surrounding air) is in progress. The combined effects of increase in droplet number density relative to this work and the presence of seeding particles tend to reduce the number of validated fringe patterns, not only in the ILIDS images but also the corresponding glare points in PIV image. Application of the present approach - including the method for eliminating the discrepancy between droplet centres on focused and defocused images, removal of glare points from the PIV image and subsequent processing of the filtered PIV images - is briefly demonstrated here for a polydisperse spray. Figure 15 presents an example of the instantaneous ILIDS and (raw) PIV images, of the PIV image after removal of glare points and of the corresponding combined droplet and gas velocity plots. The SMD of the droplets in this case was $45 \mu \mathrm{m}$. As can be observed from the velocity plot, the droplet-gas relative velocity is almost zero and - in this case - the droplets and the gas velocity vectors are closely aligned. Figure 16 shows the positions of the droplets, corresponding to the image pairs of Figure 15, obtained simultaneously from ILIDS and PIV (shown as open and blocked circles respectively). The droplet positions from ILIDS do not include the corrections for the centre discrepancy. The droplet positions from PIV are equivalent to the positions obtained from ILIDS after subtraction of corrections for centre discrepancy. As can be observed, the position of a given droplet when obtained through ILIDS differs from that obtained through PIV and the difference can be as large as $1 \mathrm{~mm}(1000 \mu \mathrm{m})$ towards the left side of the figure and it decreases towards right side. This shows that straightforward use of droplet positions obtained from ILIDS can lead to erroneous interpretation of the droplet-gas velocity spatial correlation i.e. when calculated as a function of the distance between position of the droplet and its surrounding gas velocity vectors. 

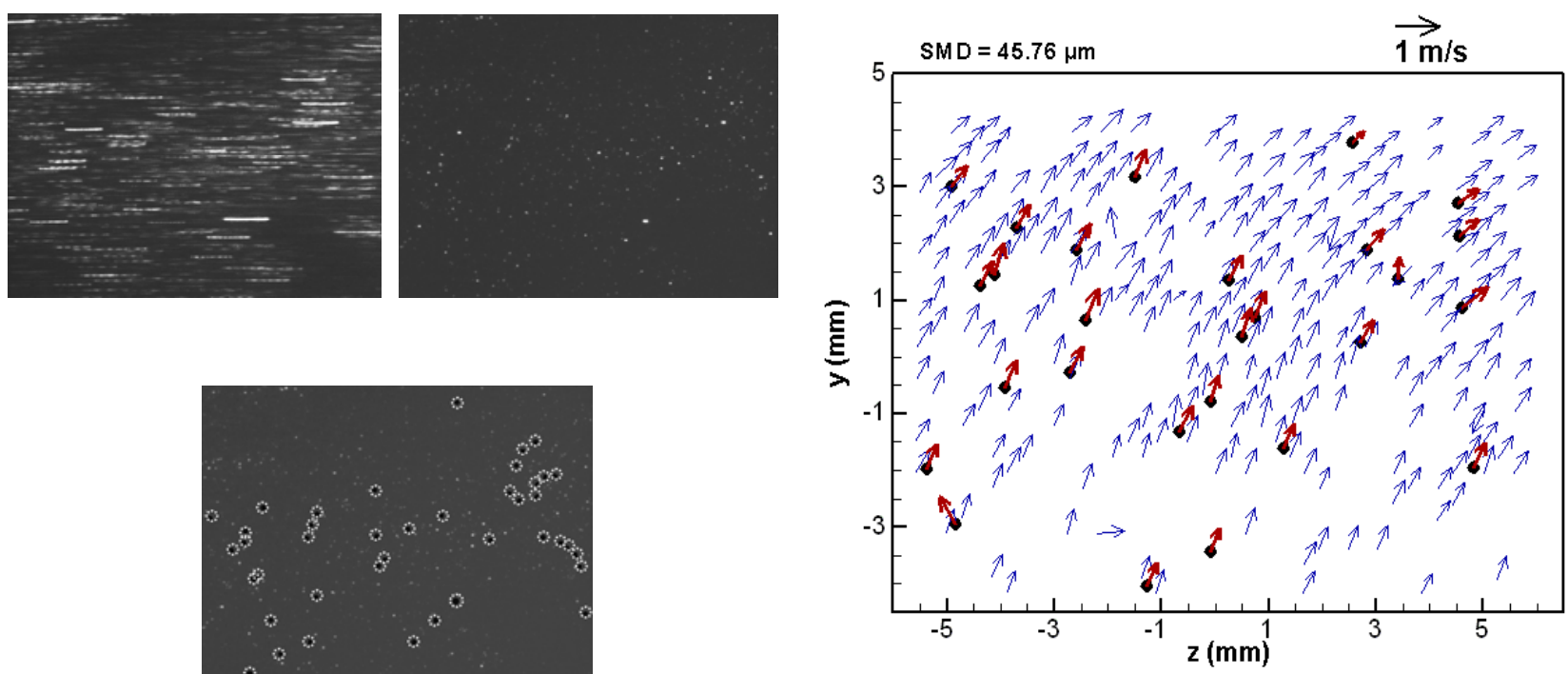

(a)

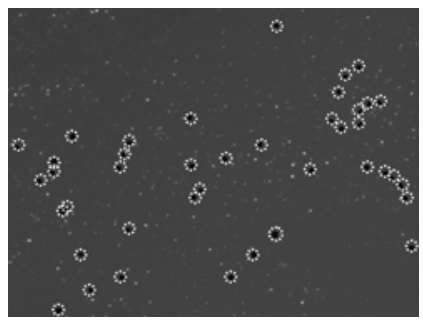

(b)

Fig. 15 (a) Instantaneous ILIDS and PIV images (top) for a polydisperse spray surrounded with seeded co flowing air and the corresponding PIV image (bottom) after removal of the glare points (the boundaries associated with the removed glare points are shown as dotted circles). (b) Simultaneous droplet and gas velocity vector plots for this case corresponding to the given time instant. Blocked circles represent droplets and the associated bold vectors represent droplet velocity

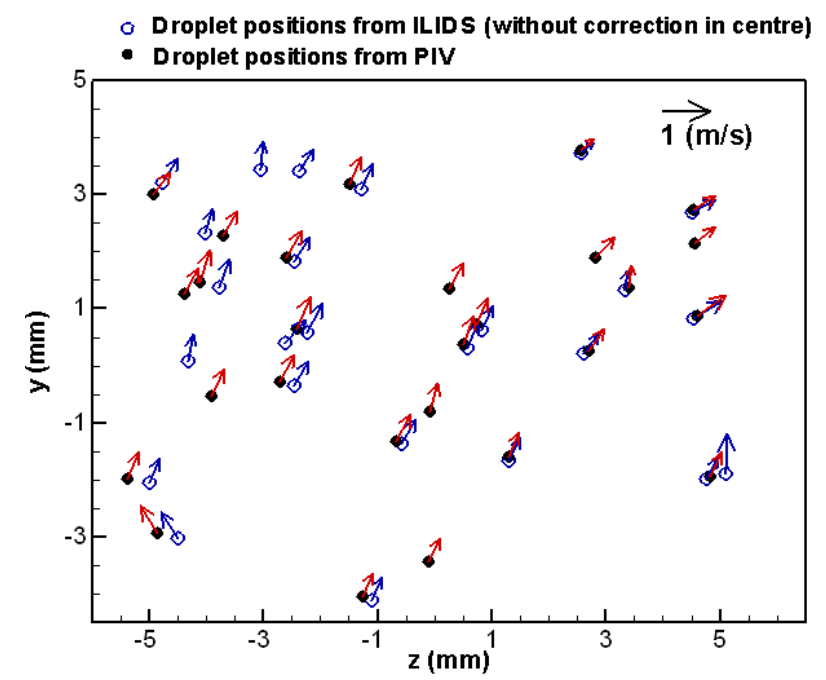

Fig. 16 Droplet positions and velocities from ILIDS (open circles) and PIV (blocked circles). Droplet positions from ILIDS are plotted without correction for centre discrepancy 


\section{Conclusions}

For the study of droplet-gas flow turbulence interaction in a spray, knowledge of droplet size is equally as important as the droplet and gas velocities. Aiming at such investigations, we have presented a new approach towards simultaneous two phase measurements in sprays by combining the out-of-focus imaging ILIDS technique with the in-focus imaging PIV technique. ILIDS provides planar droplet size and velocity, while the gas velocity in the vicinity of individual droplets is obtained by PIV. The advantage of the approach lies in its capability of identifying and removing the glare points, generated from the scattered light from droplets, from PIV images with the information of corresponding droplet position provided by ILIDS. Experiments with a stream of monosized droplets revealed that the defocusing of the ILIDS technique leads to a discrepancy between the centre location of the given droplet on the defocused and focused images. This can lead to erroneous removal of droplets or even 'seeding' particles from PIV images during the attempt to measure the gas phase velocity. An analysis was performed by considering a simple optical configuration. Both the theory and measurements show that the discrepancy varies almost linearly with the position in object plane for a given degree of defocus and can be as large as $1000 \mu \mathrm{m}$. The estimated discrepancy, obtained from experiments with a droplet generator, can be subtracted from the centre of fringe patterns in the ILIDS images, from a polydisperse spray. This approach was shown to reduce the discrepancy from about $1000 \mu \mathrm{m}$ to about $100 \mu \mathrm{m}$ and thus significantly enhance the probability of correctly identifying corresponding pairs of fringe patterns and glare points in a polydisperse spray, without seeding. The remaining discrepancy was found to be mostly due to inaccuracy in locating the centre of the fringe pattern during ILIDS processing. Simultaneous ILIDS and PIV measurements and results are reported for a monodispersed droplet stream with air, 'seeded' with particles, flowing around it. Finally an example of a polydisperse spray case 'with seeding' in air is shown to demonstrate the applicability of the technique. Also the position and velocity plots of 'only' droplets from a spray, obtained simultaneously through ILIDS and PIV, are shown, demonstrating the importance of corrections for centre discrepancy. It is concluded that after elimination of the droplet centre discrepancy, the combined ILIDS-PIV technique can be applied in a spray for simultaneous two phase measurements. Further research is being conducted to quantify the droplet concentration limit in the spray up to which the combined technique can be successfully applied. 


\section{References}

Damaschke N, Nobach H, Nonn TI, Semidetonv N, Tropea A (2005) Multi-dimensional particle sizing techniques. Exp Fluids 39:336-350

Glover AR, Skippon SM, Boyle RD (1995) Interferometric laser imaging for droplet sizing: A method for droplet-size measurement in sparse spray systems. Appl Opt 34:8409-8421

Golombokyz M, Morinyand V, Mounaim-Rousselle C (1998) Droplet diameter and the interference fringes between reflected and refracted light. J. Phys. D: Appl. Phys. 31:59-62

Hardalupas Y, Horender S (2003) A method to estimate gas-droplet velocity cross correlations in sprays. Atomization Spray 13:273-295

Kawaguchi T, Akasaka Y, Maeda M (2002) Size measurements of droplets and bubbles by advanced interferometric laser imaging technique. Meas Sci Technol 13:308-316

Kavounides C (2006) Particle Flows in Spray Dryers. PhD Thesis, Imperial College London.

Kosiwczuk W, Cessou A, Trinite M, Lecordier B (2005) Simultaneous velocity field measurements in two-phase flows for turbulent mixing of sprays by means of two-phase PIV. Exp Fluids 39: 895-908

Kurosawa R, Hishida K and Maeda M (2002) Combined measurement of LIF and ILIDS for vapour concentration and droplets size and velocity in a spray. 11th Int Symp on Applications of Laser Techniques to Fluid Mechanics Lisbon, Portugal, 8-11 July

Lindken R, Merzkirch W (2002) A novel PIV technique for measurements in multiphase flows and its application to twophase bubbly flows. Exp Fluids 33: 814- 825

Maeda M, Akasaka Y, Kawaguchi T (2002) Improvements of the interferometric technique for simultaneous measurement of droplet size and velocity vector field and its application to a transient spray. Exp Fluids 33:125-134

Palero V, Arroyo M.P, Soria J (2007) Digital holography for micro droplet diagnostics. Exp Fluids 43: 185 - 195

Palero V, Lobera J, Arroyo M.P (2005) Digital image plane holography (DIPH) for two-phase flow diagnostics in multiple planes. Exp Fluids 39: 397- 406 
Pergamalis H (2002) Droplet impingement onto quiescent and moving liquid surfaces. PhD Thesis, Imperial College London

Poelma C, Westerweel J, Ooms G (2006) Turbulence statistics from optical whole field measurements in particle-laden turbulence. Exp Fluids, 40:347-363

Rottenkolber G, Gindele J, Raposo J, Dullenkopf K, Hentschel W, Wittig S, Spicher U, Merzkirch W (2002) Spray analysis of a gasoline direct injector by means of two-phase PIV. Exp Fluids 32: 710-721

Sanchez ML, Castro F, Tinaout FV, Melgar A (2000) Considerations on the Gas-Phase Velocity Field in a non evaporating Diesel Spray. Atomization and Sprays 10: 529-543

Sugimoto D, Zarogoulidis K, Kawaguchi T, Matsuura K, Hardalupas Y, Taylor AMKP, Hishida K (2006) Extension of the compressed interferometric particle sizing technique for three-component velocity measurements. 13th Int Symp on Applications of Laser Techniques to Fluid Mechanics Lisbon, Portugal, 26-29 June

Torrence C, Compo GP (1998) A practical guide to wavelet analysis. Bulletin of the American Meteorological Society 79 : $61-78$

Zimmer L., Domann R., Hardalupas Y., Ikeda Y. (2003) Simultaneous Laser Induced Fluorescence and Mie scattering for droplet cluster measurements. AIAA J. 41, 2170-2178

\section{List of Figures}

Figure 1 (a) Principle of the combined ILIDS and PIV technique (b) Experimental set-up

Figure 2 (a) Schematic of the optical system for theoretical prediction of discrepancy in droplet centres between the focused and defocused planes. (b) Images along the axis of the lens of a droplet in the focussed and defocused planes and the corresponding coordinate systems. (c) A simplified schematic of the same optical system shown in Fig $2 \mathrm{a}$ (but with the laser sheet, focused plane and defocused plane normal to the lens axis) in order to depict the dependency of the centre discrepancy on the droplet position.

Figure 3 (a) Deviation of the geometric centre from the derived centre of the droplet image as a function of $z$ position in the object plane. (b) Discrepancy in derived centre and geometrical centre between in-focus and defocus planes as a function of $z$ position in the object plane. (c) Relative change in centre discrepancy as a function of position $x$ in the object plane for a droplet situated at three positions $z=-7,0$ and $7 \mathrm{~mm}$ respectively. (d) Comparison of the centre discrepancy for two droplet sizes of $200 \mu \mathrm{m}$ and $20 \mu \mathrm{m}$ as a function of position $z$. Focal length was $135 \mathrm{~mm}$, Magnification was 0.6 and Aperture size was $40 \mathrm{~mm}$. 
Figure 4 Simultaneous defocused (left) and focused (right) images of droplets generated by a monodisperse droplet generator (droplets flow downward)

Figure 5 (a) A typical intensity variation for a fringe pattern in the ILIDS image of a droplet and its corresponding Continuous Wavelet Transform spectrum at two different scales (left), (b) Intensity distribution of the Glare points in the PIV image corresponding to the droplets in Fig 4 and its corresponding wavelet transform spectrum and (c) Intensity distribution and wavelet transform spectrum of the glare points corresponding to the polydisperse spray.

Figure 6 Fringe patterns and corresponding glare points for a stream of monodispersed droplets without gas phase seeding at three different positions in the object plane, $z=-5.0,+1.0$ and $+5.0 \mathrm{~mm}$ respectively. The ILIDS images are superimposed on the PIV image. Note that the origin of the coordinate system $(z=0$ and $y=0)$ lies in the centre of the CCD array, which coincides with the centre of the image of the figure.

Figure 7 (a) Centre discrepancy between ILIDS and PIV images in the horizontal ( $z$ ) direction as a function of $z$ position in the object plane. The straight line is a linear fit to the original data. (b) The remaining droplet centre discrepancy after subtracting the estimated error of Fig. 7a. (c) Centre discrepancy in the vertical ( $y$ ) direction as a function of $y$ position in the object plane and the estimated error. The straight line is a linear fit to the original data. (d) The remaining droplet centre discrepancy after subtracting the estimated error of Fig. 7c

Figure 8 Simultaneous ILIDS (left) and PIV (right) images of droplets in a polydisperse spray without seeding particles

Figure 9 (a) Droplet centre discrepancy in horizontal direction $(z)$ as a function of $z$ position in the object plane for the spray. The discrepancy was obtained after subtracting the estimated error (resulting from linear fit of the measured discrepancy for the monodisperse droplet stream) from droplet centres in the ILIDS image. (b) The same information as Fig. 9a without correction. (c) Droplet centre discrepancy in the vertical direction (y) as a function of $y$ position in the object plane. The discrepancy was obtained after subtracting the estimated error from droplet centres in the ILIDS image (d) The same information as Fig. 9c without correction

Figure 10 (a) Remaining droplet centre discrepancy in horizontal direction (z) as a function of droplet size. (b) Remaining droplet centre discrepancy in vertical direction $(y)$ as a function of droplet sizes

Figure 11 Simultaneous ILIDS (left) and PIV (right) images corresponding to the monodispersed droplet stream surrounded with co flowing air seeded with aluminium oxide particles

Figure 12 (a) Demonstration of identification of glare points in PIV image using the wavelet transform. (b) The PIV image after removal of the glare points (the boundaries of the removed glare points are shown as dotted circles)

Figure 13 Flow chart for measurements of instantaneous droplet size and both droplet and gas velocity from simultaneously recorded individual pairs of ILIDS and PIV images

Figure 14 Simultaneous droplet and gas velocity vector plots for the case of the monosized droplet stream surrounded with seeding particles at a given instant. Bold vectors represent droplet velocity which is scaled down by a factor of 5

Figure 15 (a) Instantaneous ILIDS and PIV images (top) for the spray surrounded with seeded co flowing air and the PIV image (bottom) after removal of the glare points (boundaries of the removed glare points are shown as dotted circles). (b) 
Simultaneous droplet and gas velocity vector plots for this case corresponding to the given time instant. Blocked circles represent droplets and the associated bold vectors represent droplet velocity

Figure 16 Droplet positions and velocities from ILIDS (open circles) and PIV (blocked circles). Droplet positions from ILIDS are plotted with out correction for centre discrepancy 